Les ANNALES Les Annales de droit

DE DROIT

$10 \mid 2016$

Varia

\title{
Les collectivités territoriales et leurs musées dans le cadre de la loi relative aux musées de France
}

Local authorities and their museums under the law on the museums of France

\section{Claire Bosseboeuf}

\section{(2) OpenEdition}

\section{Journals}

Édition électronique

URL : http://journals.openedition.org/add/330

DOI : $10.4000 /$ add.330

ISSN : 2606-1988

Éditeur

Presses universitaires de Rouen et du Havre

Édition imprimée

Date de publication : 1 juin 2016

Pagination : $33-76$

ISBN : 979-10-240-0599-7

ISSN : 1955-0855

Référence électronique

Claire Bosseboeuf, «Les collectivités territoriales et leurs musées dans le cadre de la loi relative aux musées de France », Les Annales de droit [En ligne], 10 | 2016, mis en ligne le 08 janvier 2018, consulté le 10 décembre 2020. URL : http://journals.openedition.org/add/330 ; DOI : https://doi.org/10.4000/ add. 330

Presses universitaires de Rouen et du Havre 


\section{Les collectivités territoriales et leurs musées dans le cadre de la loi relative aux musées de France*}

\section{Claire Bosseboeuf}

La loi relative aux musées de France promulguée le 4 janvier $2002^{1}$ a posé une définition juridique du musée, et surtout balisé les contours d'un contrôle scientifique et technique de l'État sur les musées de collectivités territoriales, illustrant ainsi, en matière de musées, le principe de libre administration des collectivités territoriales. Elle consacre également l'existence d'un «recadrage» de l'État sur les musées de collectivités territoriales, à propos duquel il faut se demander s'il est toujours «à la hauteur».

L'article 72 de la Constitution de 1958 présente les collectivités territoriales comme étant les communes, les départements et les régions, les collectivités territoriales à statut particulier et les collectivités d'outremer régies par l'article 74. De fait, il n'y a pas de définition juridique des collectivités territoriales, car le texte suprême se contente d'en rappeler l'existence. Cependant les dispositions de la charte du Conseil de l'Europe sur l'autonomie locale ${ }^{2}$ permettent d'avancer qu'une collectivité territoriale est un "groupement humain, uni par un lien territorial et doté d'une organisation lui permettant de participer à la vie juridique ${ }^{3}$ ". Cette définition traduit donc la reconnaissance, à côté de l'État, de personnes morales de droit public et dotées d'attributions spécifiques.

* Cet article reprend une conférence dispensée à la faculté de droit, sciences économiques et gestion de Rouen le 18 avril 2013 dans le cycle " Histoire, patrimoine et droit » organisé par le CUREJ et dirigé par les professeurs Virginie Lemonnier-Lesage et Gilduin Davy.

1. L. $\mathrm{n}^{\mathrm{O}}$ 2002-5, 4 janv. 2002 relative aux musées de France, $J O 5$ janvier 2002, p. 305.

2. La charte de l'autonomie locale (STCE $\mathrm{n}^{0}{ }_{122}$ ), adoptée le 15 octobre 1985 a été ratifiée par la France le 17 janvier 2007.

3. Jean-Claude Douence, "Le statut constitutionnel des collectivités territoriales", Encyclopédie des collectivités locales, Paris, Dalloz, 2005, fasc. $n^{\circ} 63, \S 31$. 
Elle caractérise également la décentralisation qui marque la structure organisationnelle de la République.

Les collectivités territoriales existent à côté de l'État, mais non en dehors de lui. Aussi leur liberté d'action leur est reconnue par la Constitution de 1958 dans le cadre du principe de libre administration, caractérisé par l'existence de conseils élus dotés d'attributions effectives et de moyens juridiques et financiers propres à assurer leurs compétences. Il ne s'agit pas toutefois d'une liberté d'action pleine et entière des collectivités territoriales, mais d'une autonomie de gestion qui s'exerce dans le cadre fixé par la loi. D'où l'existence de limites, normatives ou financières. Ainsi, c'est toujours dans un cadre prévu par la loi que les collectivités territoriales exercent leurs compétences: la loi, paradoxalement, protège et encadre le principe de libre administration. Ce dernier constitue l'essence même de toute intervention locale, tel que le secteur culturel. Mais dans ce domaine, leurs compétences sont moins déterminées par la loi que par la clause générale de compétence. En effet, si l'article $1^{\text {er }}$ de la loi du 7 janvier 1983 ouvre le champ culturel à l'intervention des trois niveaux de collectivités territoriales ${ }^{4}$, leurs compétences d'attribution se limitent, dans les textes, à quelques interventions obligatoires (gestion des services d'archives ou des bibliothèques par les départements, la gestion de l'inventaire général du patrimoine culturel par les régions) et quelques initiatives permises (gestion de certains enseignements artistiques spécifiques, ou de monuments historiques nationaux sur transfert de l'État $\left.{ }^{5}\right)$. Les collectivités territoriales ont donc elles-mêmes comblé ce vide, et de fait, il n’a été " nié par personne que les collectivités territoriales pouvaient intervenir dans le domaine culturel ${ }^{6} »$. Cette intervention n'est pas contestée avant même les premières lois de décentralisation et complexifie la notion de décentralisation culturelle. Elle repose majoritairement sur la clause générale de compétence ${ }^{7}$, et permet aux collectivités territoriales de se

4. Art. $1^{\text {er }}, \mathrm{L} . \mathrm{n}^{\mathrm{o}} 83-8,7$ janv. 1983 relative à la répartition des compétences entres les communes, les départements et les régions de l'État, $J O 9$ janvier 1983, p. 215, codifié à l'article L. 1111-2 du code général des collectivités territoriales.

5. Pierre-Laurent Frier, «La répartition actuelle des compétences entre l'État et le pouvoir local », AJDA 2000, p. 60.

6. Jean-Marie Pontier, "Les interventions culturelles des collectivités territoriales", Encyclopédie des collectivités locales, Paris, Dalloz, 2009, fasc. $n^{\circ} 4170, \$ 5$.

7. Procédé "complémentaire » et "recognitif» de répartition des compétences entre l'État et les collectivités territoriales, la clause générale de compétence a été supprimée, pour les départements et les régions, par la loi $\mathrm{n}^{\circ}$ 2010-1563 du 16 décembre 2010 de réforme des collectivités territoriales (JO 18 décembre 2010, p. 22289). Restaurée par la loi $n^{0} 2014-58$ de modernisation de l'action 
présenter comme les "principaux bailleurs de fonds culturel ${ }^{8}$ ». En 2010, ces dernières ont dépensé près de 7,6 milliards d'euros pour la culture, dans des domaines variés qui touchent à l'expression artistique, à la conservation et à la diffusion du patrimoine : archives, bibliothèques et musées ${ }^{9}$. Quant à ces derniers, ils entretiennent avec les collectivités territoriales des rapports particuliers. Le nombre même de ces institutions muséales peut constituer une illustration de ces rapports: on compte environ 1300 musées répartis sur tout le territoire, dont $85 \%$ de musées publics ${ }^{10}$. Sur ces $85 \%$, «seuls " 70 appartiennent à l'État, ce qui implique que les collectivités territoriales gèrent à elles-seules près de 1000 musées, c'est-à-dire plus de 1000 " collections », car la collection constitue aujourd'hui l'essentiel du «musée ».

L'article $1^{\text {er }}$ de la loi relative aux musées de France définit le musée comme une « collection permanente composée de biens dont la conservation et la préservation revêtent un intérêt public, et organisée en vue de la connaissance, de l'éducation, et du plaisir du public». Cette définition pose les quatre caractéristiques fondamentales du musée.

En premier lieu, la spécificité du musée est telle que c'est la collection toute entière qui le constitue, et non les objets pris dans leur propre individualité. Il s'agit donc d'un ensemble de biens dont la réunion présente un intérêt qui n'est pas la seule somme des intérêts respectifs de chacun des éléments dont il est composé ${ }^{11}$.

En second lieu, cette collection doit avoir un caractère permanent. Ce caractère implique l'exclusion des centres d'art contemporains et

publique locale et d'affirmation des métropoles du 27 janvier 2014 (JO 28 janvier 2014, p. 1562), elle voit son existence de nouveau menacée par le projet de loi portant nouvelle organisation territoriale de la République, actuellement à l'étude au Parlement (Marylise Lebranchu, projet de loi $n^{0}$ 636, Sénat, session ordinaire 2013-2014). Mais, comme en 2010, il semble que la culture soit toujours épargnée par cette suppression: "les interventions multiples y sont la règle et il serait sans doute trop complexe, et trop nocif pour les activités et les acteurs [...], de vouloir y rigidifier les interventions et des financements » (Jean-Patrick Courtois, Rapport sur le projet de loi de réforme des collectivités territoriales, Sénat, session ordinaire 2009-2010, rapport $\mathrm{n}^{\mathrm{o}} 559$, p. 108).

8. Pierre-Laurent Frier, "La répartition actuelle des compétences entre l'État et le pouvoir local», art.-cité, p. 58.

9. Jean-Cédric Delvainquière, Bruno Tugorès, Nicolas Laroche et Benoît Jourdan, "Les dépenses culturelles des collectivités territoriales en 2010: 7,6 milliards d'euros pour la culture», Culture chiffres, $\mathrm{n}^{\circ}$ 3, 2014, p. 22.

10. Cours des comptes, Les musées nationaux et les collections nationales d'œuvres d'art. Rapport public particulier, 1997, p. 3.

11. Guillaume Wolkowitsch, Archives, bibliothèques, musées: statut des collections accessibles au public, Paris, Economica, 1986, p. 16-24. 
des centres d'expositions de la catégorie des musées : ainsi, le Palais de Tokyo, l'établissement public Le 104 à Paris, ou l'abbaye de Jumièges ne sont pas des musées. Il en est de même pour les centres d'expositions, dont, en France, les galeries nationales du Grand Palais à Paris ou le musée du Luxembourg sont les principaux représentants ${ }^{12}$. Sont également exclus de la catégorie «musée » les centres d'interprétation, lieux de sensibilisation, ayant vocation à donner les clés de lecture d'un site, naturel, culturel ou urbain, et dont l'intention première n'est pas de montrer des objets, mais de solliciter la sensibilité des visiteurs et de faire passer un message, de communiquer des contenus. La Corderie royale de Rochefort, par exemple, est un centre d'interprétation.

En troisième lieu, la préservation et la conservation de la collection présentent un intérêt public. Ce principe réaffirme ici le caractère génétique du musée ${ }^{13}$, sa vocation de conservatoire des œuvres qui présentent un intérêt d'un point de vue de l'histoire, de l'art, de l'archéologie, de l'esthétique, de la science ou de la technique ${ }^{14}$. Cette fonction essentielle du musée existe depuis son origine, et a été exacerbée à la Révolution : l'abolition des privilèges le 4 août 1789 , suivie de la nationalisation des biens du clergé ${ }^{15}$, des émigrés ${ }^{16}$ et de la Couronne ${ }^{17}$ ont entraîné de multiples confiscations qui marquent le début d'une période confuse, à l'origine des concepts de conservation et de protection du patrimoine.

Si les premières confiscations étaient destinées à être vendues afin de combler le déficit public, l'Assemblée législative a adopté, après la journée du 10 août 1792 et la prise des Tuileries, un décret qui proclame que «les principes sacrés de la liberté et de l'égalité ne permettent point de laisser plus longtemps sous les yeux du peuple français les monuments élevés à l'orgueil, au préjugés et à la tyrannie ${ }^{18}{ }$. S'ensuivent alors de multiples destructions massives et diverses crises iconoclastes qui se poursuivent jusqu'en 1793. En parallèle, se développe l'idée selon laquelle l'État doit se faire conservateur, afin de protéger ce qui

12. André Gob, Le musée, une institution dépassée?, Paris, Armand Colin, 2010, p. 118.

13. Étienne Fatôme, "Les musées et l'idée de service public ", dans Édouard Bonnefous, Éric Peuchot et Laurent Richer (dir.), Droit au musée, droits des musées, Paris, Dalloz, 1994, p. 15-42.

14. Art. L. 1 du code du patrimoine, qui définit ainsi le patrimoine mobilier ou immobilier.

15. D., 2 nov. 1789.

16. D., 9 nov. 1791.

17. D., 10 août 1792 .

18. D., 14 août 1792. 
risque d'être ainsi détruit: c'est à cette époque qu'apparaît le terme de "vandalisme ", dont on attribue la paternité à l'abbé Grégoire, qui a rédigé et présenté à la convention en 1794 un Rapport sur le vandalisme et les moyens de le réprimer ("j'ai créé le mot pour tuer la chose»). La nécessité de préserver le patrimoine de la destruction intervient d'abord au nom de l'instruction publique, puis progressivement au nom d'une nouvelle idéologie selon laquelle la République doit conserver des traces de son passé, car ce serait revenir à la barbarie que de l'effacer. Diverses mesures avaient déjà été prises depuis 1789 , on retiendra l'instruction de l'an II sur la manière d'inventorier et de conserver, adressée aux administrateurs de la République. Elle marque la fin des vagues de destruction et développe l'idée d'une appropriation collective des biens de la nation: «Vous n'êtes que les dépositaires d'un bien dont la grande famille a le droit de vous demander des comptes ${ }^{19}$. "

Ainsi, les multiples dépôts révolutionnaires ont logiquement concrétisé le musée, et en 1793, un décret pose le principe du transfert «au musée le plus voisin » " des monuments publics transportables, intéressant les arts ou l'histoire, qui portent quelques-uns des signes proscrits, qu'on ne pourrait faire disparaître sans leur causer un dommage réel [...] pour l'instruction nationale ». La convention crée le 27 septembre 1792 le Muséum central des arts, futur musée du Louvre, qui ouvre ses portes le 10 août 1793 .

Enfin, en quatrième lieu, la mission du musée est axée sur la connaissance, l'éducation et le plaisir du public. Là encore, ce principe fait référence à l'origine même du musée français, destiné en premier lieu à éduquer les artistes : la notion de musée naît dans la seconde moitié du XVIII ${ }^{\mathrm{e}}$ siècle, au moment de l'avènement du néoclassicisme. La volonté de renouer avec la pureté de l'art antique conduit à remettre en cause les manifestations artistiques de l'époque, et la participation de l'État dans la formation des artistes. Des intellectuels tels qu'Étienne La Font de Saint-Yenne (1688-1771) proposent d'ouvrir les collections royales au public, et de les installer au Louvre. Aussi, entre 1750 et 1779 , Louis XV permet-il l'ouverture d'un musée dans les salles du palais du Luxembourg. Outre la galerie Médicis, celui-ci présente un ensemble de 122 toiles des écoles diverses des $\mathrm{XVI}^{\mathrm{e}}, \mathrm{XVII}^{\mathrm{e}}$ et $\mathrm{XVIII}^{\mathrm{e}}$ siècles, qui ont

19. Instruction sur la manière d'inventorier et de conserver, dans toute l'étendue de la République, tous les objets qui peuvent servir aux arts, aux sciences et à l'enseignement, proposée par la commission temporaire des arts et adoptée par le Comité d'instruction publique de la Convention nationale du 25 ventôse an II (15 mars 1794). 
vocation à servir de manifestes et à contribuer à la formation des artistes. Dès 1763 , il était question de transférer ces œuvres au Louvre.

Ces critères de définition du musée permettent de conclure à sa vocation de service public. L'analogie que pose la loi du 4 janvier 2002 relative aux musées de France avec la connaissance et l'éducation, ainsi qu'avec l'idée de conservation qui découle du concept de patrimoine national développé durant la Révolution, implique que le musée relève de la catégorie des services publics administratifs. Il s'agit d'un aspect essentiel du musée dont il ne parait pas pertinent de se détacher ${ }^{20}$, et qui se pose comme la consécration du fait que l'État et les collectivités territoriales restent garants de la diffusion culturelle et de la qualité du service.

Quant aux musées des collectivités territoriales, il est communément admis que leur naissance remonte à l'arrêté Chaptal du $1^{\text {er }}$ septembre 1801 (14 fructidor an IX). Ce ministre de Napoléon Bonaparte a en effet établit par arrêté une liste de quinze villes réparties sur l'ensemble des territoires de la République; chacune d'elle étant destinée à accueillir un musée de beaux-arts dont les collections devaient être constituées des dépôts révolutionnaires et des envois du pouvoir central liés aux conquêtes napoléoniennes ${ }^{21}$. Cet acte illustre la volonté de l'État de s'investir en matière de musées, et marque le début d'un contrôle national sur les institutions locales. Il est le point de départ de l'organisation progressive des relations entre l'État et les collectivités territoriales, qui ont trouvé un aboutissement au moment de la décentralisation et surtout avec la loi du 4 janvier 2002 relative aux musées de France (1). Promoteur d'une certaine qualité culturelle, cette dernière impose aux musées dotés de l'appellation «musée de France » un certain nombre de contraintes liées à la gestion de leurs collections. Celles-ci suscitent cependant un certain nombre d'interrogations, liées justement à la nécessité de concilier libre administration des collectivités territoriales et contrôle de l'État (2).

\section{Aperçu de l'histoire des musées territoriaux et de l'évolution de leurs relations avec l'État}

S'en tenir au seul arrêté Chaptal pour parler de l'histoire des musées territoriaux serait un peu réducteur, et reviendrait à nier les multiples initiatives des collectivités territoriales en matière de musées, qui se

20. Jacques Sallois (intervention sous Étienne Fatôme), «Les musées et l'idée de service public », dans Édouard Bonnefous, Éric Peuchot et Laurent Richer (dir.), op. cit., p. 36.

21. Cet arrêté concernait les villes de Bordeaux, Bruxelles, Caen, Dijon, Genève, Lille, Lyon, Marseille, Mayence, Nancy, Nantes, Rouen, Rennes et Strasbourg. 
sont affirmées très tôt dans l'histoire. Ces dernières ont parfois même « devancé » l'État, à tel point qu'aujourd'hui le territoire est " couvert » de musées territoriaux, comme il a pu l'être d'églises au début du deuxième millénaire (1.1). L'histoire des musées territoriaux est cependant fortement liée au développement des relations qu'ils ont entretenues avec l'État. La politique interventionniste mené par ce dernier dès la Révolution a fini par trouver un aboutissement avec l'acte I de la décentralisation et, surtout, la loi du 4 janvier 2002 relative aux musées de France (1.2).

\subsection{Le fort investissement des collectivités territoriales dans le développement des musées locaux}

$\mathrm{Au} \mathrm{XVIII}{ }^{\mathrm{e}}$ siècle, les théories et principes développés sur l'ouverture des collections royales au public trouvent rapidement un écho au niveau local, et l'on peut relever très tôt l'organisation de la publicité des collections particulières, par le biais de journaux, guides de voyages et autres almanachs. On doit également mentionner un certain nombre d'initiatives novatrices des collectivités territoriales en matière de musées, et une forte volonté de ces dernières de créer, dès la Révolution, leur propre institution. Ce « désir» de musée a perduré tout au long du $\mathrm{xx}^{\mathrm{e}}$ siècle, et a été marqué par une véritable frénésie de construction.

Ainsi, si la Révolution française est prête à accueillir le musée, c'est grâce à de multiples initiatives novatrices qui ont préparé son avènement, que ce soit au niveau central ou au niveau local. La "province" a fait preuve d'imagination dans ce domaine, et son intervention a pu prendre au moins trois formes: l'ouverture des collections au public dans le cadre des dons et legs, la volonté de mettre en avant l'héritage historique du territoire, et la création d'écoles d'art.

Les collectivités territoriales ont été les premières personnes publiques à former des "musées » en France, constitués des collections d'érudits qui ont très tôt pris la mesure de l'importance de celles-ci. Dès lors, ils ont souhaité les mettre à la disposition du public. L'exemple le plus ancien serait le legs de l'abbé Jean-Baptiste Boisot au couvent bénédictin de Besançon en 1694. Celui-ci aurait reconstitué en partie et à ses frais la collection de peintures et de sculptures de deux notables de la région. Jean-Baptiste Boisot a assorti son legs de la condition expresse que le couvent en organise la maintenance et l'accès libre et gratuit au public au moins deux fois par semaine. Cet exemple, le premier en son genre, n'est pas isolé et il a contribué a entériné la pratique des dons et legs, 
procédure courante, devenue, au $\mathrm{XIX}^{\mathrm{e}}$ siècle, une « $\operatorname{tradition}^{22}$ » : le musée peut alors naître grâce à une donation qui constitue le noyau initial de la collection (legs du cabinet de curiosité de l'abbé Favre à la ville d'Annecy en 1840), ou contribue à l'enrichissement et à la dynamisation d'une collection existante, telle qu'un dépôt révolutionnaire tombé lentement dans l'oubli (comme à Quimper, Nantes, Villars ou Aix-en-Provence).

Le musée est également lié à une politique de développement de l'attractivité du territoire, très novatrice et précurseur, dans les villes marquées par la présence de vestiges des civilisations antiques. Par exemple, dès la fin du $\mathrm{XvII}^{\mathrm{e}}$ siècle, la commune d'Arles, associée aux autorités religieuses s'est préoccupée de la conservation des nombreux vestiges gallo-romains qui parsèment son territoire. En 1784, elle a ainsi créé le "Museum Arelatense », constitué d'un dépôt de ces vestiges dans la cour du couvent Saint-Honoras.

Enfin, l'émergence, à partir du XVIII ${ }^{\mathrm{e}}$ siècle, des écoles d'art sur tout le territoire est un facteur important du développement des musées territoriaux. Cette époque est marquée par une nouvelle conception de l'enseignement et de la pédagogie, qui a particulièrement concerné les arts et les techniques. Dès 1740, les académies se sont intéressées à l'enseignement du dessin, devenu essentiel à la formation des artistes et des artisans. Deux textes ont développé et diffusé ces théories relatives à l'éducation de la main et du regard, respectivement écrits par Antoine Ferrand de Monthelon ${ }^{23}$ et Jean-Baptiste Descamps ${ }^{24}$; ils sont tous deux à l'origine de la constitution d'une école de dessin à Rouen et à Reims. Ces écoles se sont multipliées sur le territoire, notamment à Lyon, Marseille, Dijon, Orléans, et ont préfiguré le musée : l'éducation passant par l'observation des œuvres, elle impliquait la constitution d'un fonds de modèles (peintures, dessins, sculptures, qui pouvaient être des copies ou des moulages). Les productions des élèves étaient conservées, et portaient principalement sur des sujets relatant l'histoire locale. Elles sont, dans bien des cas, à l'origine de la constitution des collections d'un musée (la ville de Dijon avait mis en place un Prix de Rome, et les envois des élèves s'intégraient dans les collections muséales). Par ailleurs, elles

22. Chantal Georgel, «Collection privées/collections publiques: un dialogue permanent », Cahiers du GRHIS, n ${ }^{\circ}$ 4, 1996, p. 23-30.

23. Antoine Ferrand de Monthelon, «Projet pour l'établissement d'écoles gratuites de dessin, suivi de la réponse du Révérend Père Castel », Mercure de France, mars 1746, $4 \mathrm{p}$.

24. Jean-Baptiste Descamps, Sur l'utilité des établissements des écoles gratuites de dessin en faveur des métiers, Paris, Regnard, 1767, 48 p. 
étaient à la base d'un lien avec le public, les productions des élèves et leurs modèles faisant en effet l'objet d'expositions.

Fortes de ces expériences et marquées par les idées révolutionnaires fondées sur la conservation des œuvres d'art qui font partie d'un héritage que la France "régénérée ne saurait répudier sans honte ${ }^{25}$ ", les collectivités territoriales ont souhaité récupérer une part des dépôts constitués dès 1789. Le 2 décembre 1790, Louis-Georges de Bréquigny présentait à la commission des monuments ${ }^{26}$ un rapport proposant une répartition équitable de ces derniers sur tout le territoire. En effet, les biens du clergé, de la noblesse et de la Couronne ayant été nationalisés, la constitution de ces dépôts en province devenait logiquement une prérogative régalienne. Cette répartition devait conduire à la création, dans les 83 départements nouvellement définis, d'un dépôt "aussi complet qu'il se pourra». Un décret du 16 septembre 1792 a entériné ce principe. Mais dans le contexte de la création du Muséum central des arts, institution à vocation universelle, il a été décidé de garder au Louvre les œuvres de "première classe », et d'envoyer les autres en province dans le cadre de dépôts, c'est-à-dire de prêts à long terme. Les communes se sont insurgées contre cette pratique, au nom de la liberté et de l'égalité. Des lettres et des pétitions ont circulé à destination du gouvernement (à Grenoble par exemple en 1798), et les municipalités ont commencé à créer, des musées à caractère "provisoires ${ }^{27}$ ». En réponse, le gouvernement n'avait pas de politique claire et précise, et agissait vis-à-vis des collectivités de manière assez opportuniste, récompensant ici ou là le patriotisme d'une municipalité, ou la punissant, au contraire, pour sa déloyauté. L'arrêté Chaptal du $1^{\text {er }}$ septembre 1801 qui institue quinze musées de province dans les grandes villes de la République n'est donc que l'aboutissement des multiples transactions qui ont eu lieu entre l'État et les communes. Il est également une réponse à la question posée par l'arrivée, en 1798, des chefs d'œuvres confisqués lors des campagnes d'Italie et à la nécessité d'assurer rapidement leur conservation.

25. D., 16 sept. 1792.

26. Créée par l'Assemblée nationale en 1790, la Commission des monuments avait pour mission d'assurer l'inventaire des richesses du patrimoine. Composée d'historiens et d'érudits, elle était présidée par Louis-Georges de Bréquigny, historien et paléographe français (1714-1795).

27. Édouard Pommier mentionne ainsi l'existence d'une quinzaine de musées de fait, à Rouen, Dijon, Grenoble, Angers, au Mans notamment (Chantal Georgel, «L'État et "ses" musées de province ou comment "concilier la liberté d'initiative des villes et les devoirs de l'État" ", Le mouvement social, n 160, 1992, p. 65-77). 
Ces tractations entre l'État et les communes se sont poursuivies tout au long du XIX ${ }^{\mathrm{e}}$ siècle, les collectivités territoriales continuant à créer des musées, à tel point qu'il a été écrit que "peu importent finalement les collections, pourvu qu'il y ait un musée ${ }^{28} »$. Celui-ci illustrait également la volonté de mettre en avant l'histoire du territoire et de lutter contre le « jacobinisme centralisateur" en laissant des traces de l'histoire du territoire. Ainsi, très vite, le musée s'inscrit comme un lieu d'identité et d'ancrage du territoire local. Cette ambition se concrétise par la mise en avant des hommes illustres et des artistes. Ce discours a perduré jusqu'au tournant $\mathrm{du} \mathrm{xx}^{\mathrm{e}}$ siècle, encouragé par les sociétés savantes, et notamment le comité scientifique des beaux-arts: "Les dieux sont au Louvre, ouvrez vos portes aux maitres provinciaux ${ }^{29}$ !» Le refus du centralisme étatique contribue à l'enrichissement des collections des musées, sous l'impulsion des sociétés savantes ou des élus locaux. Souhaitant exposer des œuvres modernes, ils envoient régulièrement leur personnel au Salon pour acquérir des œuvres : par exemple, l'acquisition par la ville de Bordeaux de La Grèce sur les ruines de Missolonghi de Delacroix en 1851, est due à la forte influence de la société savante. Enfin, parfois, la thématique des beaux-arts passe en second plan derrière l'archéologie, l'ethnologie ou l'histoire, les musées développant ainsi une nouvelle fonction éducative. De même, "un important mouvement de création d'écoles et de musées "d'art industriel", "d'art et d'industrie" ou "d'arts décoratifs" accompagne l'épanouissement de l'industrie et des métiers d'art depuis le début du XIX ${ }^{\mathrm{e}}$ siècle, tout en donnant à voir une véritable préoccupation de "moraliser" la société française, dans un climat de concurrence avec l'Angleterre».

Ces diverses manifestations du "désir de musée » des collectivités territoriales ont contribué à faire du $\mathrm{XIX}^{\mathrm{e}}$ siècle «le siècle des musées »: à l'aube $\mathrm{du} \mathrm{xx}^{\mathrm{e}}$ siècle, 600 institutions de ce type sont répertoriées dans toute la France, contre une quinzaine en $1800^{30}$.

À l'inverse, les musées au $\mathrm{xx}^{\mathrm{e}}$ siècle ont connu une évolution assez lente, marquée cependant dans l'entre-deux-guerres par l'affirmation de

28. Chantal Georgel, «Le musée lieu d'identité», dans Chantal Georgel (dir.) La jeunesse des musées, catalogue d'exposition, Paris, RMN, 1994, p. 109.

29. Henry Jouin, "L'art et la province », Le comité des sciences de beaux-arts, les sessions annuelles des délégués des départements, Orléans, 1901, cité par Chantal Georgel, "Le musée lieu d'identité », art. cité, p. 110.

30. Frédéric Poulard, Conservateurs de musées et politiques culturelles: l'impulsion territoriale, Paris, La Documentation française, 2010, p. 17-18. 
la profession de conservateur de musée ${ }^{31}$, qui s'impose progressivement et remplace l'élite locale à la tête des établissements.

Après la seconde guerre mondiale, les impératifs de reconstruction ont placé les musées au deuxième plan des politiques nationales et locales. Quelques établissements ont cependant vu le jour après 1945. À cette période, 642 villes possédaient un musée, et, entre le $1^{\mathrm{er}}$ janvier 1945 et le 31 décembre 1965, 169 nouveaux musées ont été créés, dont 117 à l'initiative des communes ${ }^{32}$. Mais ce sont surtout les années 1970 qui ont amorcé une période très favorable aux musées, marquée par l'investissement croissant des personnes publiques et surtout des collectivités territoriales dans ce domaine.

La vision du musée change et sa reconnaissance comme facteur du développement local incite à la création de partenariats entre collectivités territoriales et donc au développement des financements croisés. Par ailleurs, des institutions d'un genre nouveau naissent : les écomusées et les musées de société. Dans le cadre d'une organisation muséographique innovante, il s'agit de présenter et d'interpréter l'homme dans son milieu - naturel, industriel, urbain. Ces nouveaux musées sont nés en partie grâce à l'implication de Georges-Henri Rivière, conservateur et créateur entre 1937 et 1967 du musée des Arts et Traditions populaires de Paris ${ }^{33}$.

Enfin, les lois de décentralisation et la politique des grands travaux de l'État (avec le succès du Centre Georges-Pompidou, du musée d'Orsay et la rénovation complète du musée du Louvre) ont eu un fort impact sur les élus locaux. Aussi, nombre de collectivités territoriales se sont-elles engagées dans la création, la réfection ou l'agrandissement de musées: en 1993, la direction des musées de France mentionnait l'existence de quatre cents chantiers achevés ou en cours depuis 1981,

31. En 1920 est créée l'Association générale des conservateurs des collections publiques de France.

32. Frédéric Poulard, Conservateurs de musées et politiques culturelles, op. cit., p. 28.

33. Georges-Henri Rivière (1897-1985): conservateur de musée, sous-directeur du musée d'ethnographie du Trocadéro. En 1935, il propose la création d'un «musée français», qui ouvre ses portes en 1937 sous le nom de «musée des Arts et Traditions populaires », et dont il est le directeur jusqu'en 1967. Premier directeur du Conseil international des musées entre 1947 et 1965, il en est ensuite resté membre permanent et conseiller. Il est à l'origine du développement du concept d'écomusée, et a parcouru le territoire national et le monde pour apporter son conseil et son soutien à la constitution de multiples structures de ce type (notamment pour le musée du Vin à Beaune, le musée de la fondation Gulbenkian à Lisbonne, le musée international de la Croix-Rouge et le musée de l'Horlogerie en Suisse). 
et « la rumeur prétend qu'il s'en ouvre un tous les mois ${ }^{34}$ ». Les villes de Villeneuve-d'Ascq (1983), Bordeaux (1984), Nantes (1993), Rouen (1994), Saint-Étienne (1987), Grenoble (1994), Marseille (1994), Lille (1997) notamment, ont ouvert ou rouvert leurs musées durant cette période.

Aujourd'hui, près de mille musées sont gérés par les collectivités territoriales. Parmi ceux-ci, toutes les thématiques sont abordées : archéologie, arts décoratifs, civilisations extra-européennes, ethnologie, histoire, photographie, sciences naturelles et sciences fondamentales et bien entendu, musées de beaux-arts et d'art contemporain. On peut ainsi citer un musée de la Chemiserie et de l'Élégance masculine à Argentonsur-Creuse, un musée du Temps à Besançon, et un musée de la Lunette à Morez. La frénésie de construction des années 1980-1990 s'est apaisée, bien que l'on compte encore une quinzaine d'inaugurations par an. Le musée reste un enjeu important pour les collectivités territoriales, et de nouveaux projets sont en cours de réalisation ou viennent de s'achever, de la construction du musée des Confluences du département du Rhône à celle du nouveau musée du Louvre à Lens, en passant par un grand nombre de rénovations et de réhabilitations.

Le tableau ci-dessous présente une répartition des musées territoriaux par catégorie de collectivités territoriales ${ }^{35}$ :

Autres catégories

Municipaux Départementaux Régionaux de collectivités territoriales

Total (groupements)

\begin{tabular}{lccccc}
\hline $\begin{array}{l}\text { Nombre } \\
\text { de musées }\end{array}$ & 798 & 108 & 6 & 85 & 997 \\
\hline $\begin{array}{l}\text { Dont COM } \\
\text { et DROM }\end{array}$ & 5 & 8 & 4 & 0 & 17 \\
\hline$\%$ & 80,04 & 10,83 & 0,60 & 8,53 & 100 \\
\hline
\end{tabular}

Les communes restent majoritaires dans la gestion des musées territoriaux, devant les départements, et loin devant les régions. Cette configuration s'inscrit dans la tradition: historiquement, le musée est issu d'initiatives communales. Ce développement est sans doute dû à la réforme de la carte administrative de la France par les révolutionnaires

34. Michel Guerrin et Emmanuel de Roux, "La fièvre des musées. I. Les nouveaux temples de la consommation culturelle», Le Monde, 2 février 1993.

35. Source: département des études, de la prospective et des statistiques du ministère de la Culture, mars 2011. 
de $1789^{36}$. La loi du 14 décembre 1789 a octroyé des prérogatives importantes aux communes, en consacrant notamment dans son article 49 la double fonction qu'elles exercent encore aujourd'hui. Chargées de l'administration générale de l'État, elles disposent également de fonctions propres et la loi de 1789 annonçait la clause générale de compétence posée par la loi municipale du 5 avril $1884^{37}$ dont les départements bénéficiaient depuis la loi du 10 août $1871^{38}$. Le développement des musées territoriaux reposerait donc, au moins jusqu'en 1983, sur la consécration et la mise en œuvre, par les collectivités territoriales, de la clause générale de compétence ${ }^{39}$. Avec l'acte I de la décentralisation, les musées s'inscrivent dans les initiatives culturelles permises par le législateur aux collectivités territoriales : l'article 62 de la loi de décentralisation du 22 juillet 1983 dispose en effet que «les musées des régions, des départements et des communes sont organisés et financés par ceux-ci ${ }^{40}$ [...] \ Cette disposition est légitime et consacre une situation existant de fait, tout en reconnaissant à la région, nouvellement érigée au rang de collectivité territoriale ${ }^{41}$, la possibilité de créer également des musées. Celles-ci sont encore peu intervenues dans la création de musées, mais on peut citer quelques musées régionaux: le musée régional de Fouras (Charente-Maritime), le musée régional d'Histoire et d'Ethnographie de Fort-de-France (Martinique) et, dernièrement, la reprise par la région Languedoc-Roussillon de la gestion du musée municipal d'Art contemporain de Sérignan (Hérault ${ }^{42}$ ).

Les dispositions de l'article 62 de la loi du 22 juillet 1983, autrefois codifiées à l'article L. 1423-1 du code général des collectivités territoriales, ont été inscrites dans le code du patrimoine, dans une nouvelle rédaction issue de la loi du 4 janvier 2002 relative aux musées de France. L'article L. 410-2 de ce code dispose dorénavant que «les musées des collectivités territoriales ou de leurs groupements sont organisés et financés par la collectivité dont ils relèvent». Le législateur a ainsi

36. Les découpages de l'Ancien Régime ont ainsi été complètement remis à plat. C’est dans ce contexte notamment que sont nés les départements (D., 26 fév. 1790).

37. L., 5 avr. 1884 relative à l'organisation municipale, $J O 6$ avril 1884, p. 1557.

38. L., 10 août 1871 relative aux conseils généraux, $J O 29$ août 1871, p. 3041.

39. Art. L. 2121-29 du code général des collectivités territoriales pour les communes, art. L. 321161 pour les départements et L. 4221-1 pour les régions.

40. Art. $1^{\text {er }}$, L. $n^{\circ} 83-663,22$ juil. 1983 complétant la loi $n^{\circ} 83-8$ du 7 janvier 1983 relative à la répartition des compétences entre les communes, les départements, les régions et l'État, $J O 23$ juillet 1983, p. 2286.

41. Art. 59, L. $\mathrm{n}^{\circ}$ 82-213, 2 mars 1982 relative aux droits et libertés des communes, des départements et des régions, $\mathrm{JO}_{3}$ mars 1982, p. 730.

42. Décision du conseil municipal de Sérignan du 16 novembre 2009. 
pris en compte la naissance d'une nouvelle forme de tutelle sur les musées locaux : celle des groupements de collectivités territoriales, c'està-dire le recours à l'intercommunalité pour gérer des musées. Sont ainsi visées l'intercommunalité de projet et l'intercommunalité de service, dans le cadre, par exemple, de l'établissement public de coopération culturelle ${ }^{43}$ créé par la loi du 4 janvier 2002. Le musée de Céret, créé en 2004 par le conseil régional du Languedoc-Roussillon, le conseil général des Pyrénées-Orientales et la ville de Céret, en est l'un des premiers représentants.

Ces dispositions du code du patrimoine instituent donc légalement une compétence partagée des collectivités territoriales en matière de musées, ceux-ci relevant alors de la catégorie des services publics dits "facultatifs ", c'est-à-dire ceux dont la loi se contente de prévoir l'existence. Il revient alors aux autorités locales de décider de manière discrétionnaire de leur création ou de leur suppression.

\subsection{Les relations État/collectivités territoriales: l'affirmation du contrôle de l'État sur les collectivités territoriales en matière de musées}

Les lois de 1983 ne se sont pas contentées de "prévoir l'existence» des musées territoriaux. Elles ont également posé l'idée d'un contrôle, "contrôle technique de l'État». Fondé "sur la qualité des œuvres et sur un certain nombre de dispositions", il paraissait "indispensable si [nous voulons] que les musées de province puissent présenter des expositions intéressantes et disposer de façon permanente de tableaux et d'œuvres d'art qui le permettent». "On ne peut pas demander à un musée de province d'avoir une personnalité qualifiée pour porter des jugements ${ }^{44}$.» Ainsi, sans nier «la possibilité d'une autonomie du pouvoir de décision des autorités locales", leur politique culturelle ne peut que "s'inscrire dans le cadre de la politique culturelle de l'État parce que seul ce dernier dispose, auprès des citoyens, de la légitimité qui justifie des interventions en ce domaine ${ }^{45}$ ». Cette affirmation conduit à de multiples questionnements sur la notion même de décentralisation

43. L. $\mathrm{n}^{0} 2002-6,4$ janv. 2002 relative à la création d'établissements publics de coopération culturelle, JO 5 janvier 2002, p. 309.

44. Exposé des motifs de la loi $n^{0} 83-663$ du 22 juillet 1983 complétant la loi $n^{0} 83-8$ du 7 janvier 1983, relative à la répartition des compétences entre les communes, les départements, les régions et l'État, $J O 23$ juillet 1983, p. 2286.

45. Jean-Marie Pontier, "Les interventions culturelles des collectivités territoriales», art. cité, $\$ 9$. 
culturelle, et il semble que la culture soit subordonnée à des fins qui ne relèvent pas des collectivités territoriales mais de l'État. De ce fait, la décentralisation culturelle n'est pas la simple mise en évidence de réalités locales et il est difficile, alors, de distinguer ce qui relève de la décentralisation culturelle et ce qui relève des politiques culturelles locales $^{46}$. Il reste que la légitimité de l'État se manifeste par deux fonctions essentielles: la "labellisation », et le contrôle scientifique et technique ${ }^{47}$. Quant aux musées, la loi du 4 janvier 2002 institue une forme de labellisation avec l'appellation "musée de France ", ainsi qu'un contrôle scientifique et technique. Elle est la conclusion de multiples tentatives de l'État d'imposer son contrôle sur les musées territoriaux, qui remontent, pour les premières, à la Révolution. Elle pose un cadre juridique homogène des musées avec l'appellation «musée de France», qui ne concerne cependant que les institutions qui en font la demande.

Dès la fin du XVIII ${ }^{\mathrm{e}}$ siècle, l'État a voulu imposer son contrôle sur les musées territoriaux. Cette volonté est justifiée par le fait que, à l'issue de la nationalisation des biens confisqués au clergé, aux émigrés, aux académies et à la Couronne, leur gestion était devenue une compétence exclusive du pouvoir central. Ainsi, une circulaire du 14 mars 1791 affirmait le pouvoir de l'Assemblée constituante en matière artistique. Par la suite, ayant procédé à une répartition des richesses nationales sur tout le territoire, il paraissait normal que l'État s'assure de leur bonne conservation. Cependant, malgré ses ambitions, il s'est peu investi vis-à-vis des musées de province. Cette position a satisfait les collectivités territoriales, désireuses de s'affirmer en dehors de toute directive nationale. La situation a changé après avoir été relevée par de nombreux intellectuels. Surtout, en 1880, l'historien et critique d'art Henri Houssaye (1848-1911) publie un article dans La Revue des deux mondes, dans lequel il déplore la situation des musées de province, lesquels pourraient parfaitement vendre ou aliéner leurs collections «sans que la Direction des Beaux-Arts fût fondée à s'y opposer ${ }^{48}$ ".

Cet article a suscité de vives réactions, et a trouvé une réponse dans la circulaire du sous-secrétaire d'État aux Beaux-Arts, Édouard Turquet, du 26 avril 1881. Prenant en compte l'abandon dans lequel

46. Jean-Marie Pontier, «La décentralisation culturelle», BJCL 2008, $\mathrm{n}^{\circ}$ 1, p. 2-17.

47. Jean-Marie Pontier, "Les interventions culturelles des collectivités territoriales", art. cité, $\$ 9$.

48. Henri Houssaye, "Les musées de province, les origines et l'organisation », Revue des deux mondes, $1^{\text {er }}$ avril 1880 , p. 546-580, cité par Chantal Georgel, "L'État et "ses" musées de province...», art. cité, p. 70. 
les gouvernements précédents avaient laissé les musées de province, celui-ci préconise la présentation d'un projet de loi qui placerait les collections publiques des musées dans une situation proche de celle dans laquelle se trouvaient les bibliothèques, en application de l'ordonnance du 22 février $1839^{49}$. Cette circulaire a ouvert la voie à une avalanche de dispositions relatives à la création d'une inspection des arts du dessin et des musées (arrêté du 13 janvier 1887), à la nomination des conservateurs et la restauration des œuvres appartenant à l'État (circulaire du 14 août 1890) ou à «l'installation des musées de province» (circulaire de février $1901^{50}$ ).

Ainsi l'État, après quelques années «d'oubli », redécouvrait-il l'existence de "ses" musées de province, au nom de l'éducation et de la vocation pédagogique du musée. Or, ce regain d'intérêt a permis aux collectivités territoriales de justifier leurs demandes d'envois d'œuvres. Ces demandes, destinées à accroître leur prestige local, justifient donc un contrôle de l'État. Plusieurs textes ont ainsi été adoptés, jusqu'à l'ordonnance du 13 juillet $1945^{51}$ qui posait une distinction entre musées classés et musées contrôlés. L'absence de dispositions législatives et réglementaires précises, associée au fort développement et à la diversification des institutions muséales (et notamment des institutions locales ${ }^{52}$ ) a contribué à remettre en cause cette ordonnance qui, dans son intitulé, présentait de toutes façons un caractère "provisoire». En 1993, le ministre de l'Éducation nationale et de la Culture a soumis au Conseil des ministres un projet de loi relatif «aux musées, aux établissements publics territoriaux à vocation culturelle et aux restaurateurs du patrimoine ${ }^{53}$ ». Il proposait la suppression de l'ordonnance de 1945 et la mise en place "de principes généraux cohérents et modernes " pour l'organisation des musées. Ce projet est resté sans suite jusqu'en 2000, date à laquelle M. Alfred Recours a présenté à l'Assemblée nationale un rapport sur les musées.

49. Ordonnance royale du 22 février 1839 sur l'organisation des bibliothèques publiques. Ses dispositions prévoyaient notamment que les bibliothécaires étaient nommés par le ministre de l'Instruction publique.

50. Chantal Georgel, «L'État et "ses" musées de province... », art. cité, p. 65.

51. Ord. $n^{0} 45-1546,13$ juil. 1945, portant organisation provisoire des musées de beauxarts.

52. Frédéric Poulard, Conservateurs de musées et politiques culturelles, op. cit., p. 157.

53. Jack Lang, Projet de loi relatif aux musées, aux établissements publics territoriaux à vocation culturelle et aux conservateurs, projet $\mathrm{n}^{\circ} 202$, Sénat, session ordinaire 1992-1993. 
Il s'articule autour de trois questions :

Comment définir le musée et garantir sa qualité et son niveau d'exigence scientifique et artistique, sans pour autant l'enfermer dans un statut par trop rigide? Comment vitaliser et développer l'action en direction du public et l'ouverture vers l'extérieur, afin de donner aux musées toute leur place dans une politique de démocratisation culturelle? Comment insérer le musée dans la décentralisation et en faire un vecteur dynamique d'aménagement culturel du territoire ${ }^{54}$ ?

La loi du 4 janvier 2002 relative aux musées de France répond à ces problématiques, en consacrant l'idée d'un « label qualité » octroyé aux institutions qui en font la demande.

Cette appellation "musée de France " institue un régime juridique homogène en se substituant à toute classification antérieure. Elle illustre la volonté d'orienter le public, de lui montrer la différence entre une collection ayant un intérêt scientifique véritable et une autre, ayant surtout une vocation commerciale et n'ayant de musée que le nom. Elle n'est pourtant pas directement et explicitement définie, bien qu'elle se caractérise par rapport aux dispositions qui régissent son attribution et aux obligations qui incombent aux musées ainsi labellisés. Avec la loi du 4 janvier 2002, les régimes et classifications antérieurs des musées sont abolis. L'article 18 de la loi précise en effet que «l'appellation "musée de France" est attribuée aux musées nationaux, aux musées classés en application des lois et règlements en vigueur antérieurement à la présente loi et aux musées de l'État dont le statut est fixé par décret ${ }^{55}$ [...]». Il rappelle également que « les musées contrôlés en application des lois et règlements en vigueur antérieurement à la présente loi reçoivent l'appellation "musée de France" ".

Jusqu'en 2002 en effet, les musées étaient soumis à des réglementations différentes, le contrôle de l'État s'exerçant par le biais de la direction des musées de France ou du Muséum national d'histoire naturelle. L'origine des musées nationaux est marquée par la naissance, au XVIII ${ }^{\mathrm{e}}$ siècle, du musée du Louvre, du musée des Sciences et Techniques et du Muséum d'histoire naturelle. Ces institutions ont vite été considérées comme des institutions "pilotes", qui se devaient d'exercer, chacune dans leur domaine, un contrôle sur les musées de province. Le ministère de la Culture, par le biais de la direction des musées de France, a récupéré le contrôle sur les musées de beaux-arts, c'est-à-dire

54. Alfred Recours, Rapport d'information sur les musées, rapport $n^{\circ} 2418$, Assemblée nationale, 2000, $93 \mathrm{p}$.

55. Art. L. 442-2 du code du patrimoine. 
ceux qu'il jugeait dignes d'intérêt et qui figuraient sur une liste dite de musées « classés ${ }^{56}$ ». Une partie de ceux-ci était issue des quinze musées de province institués par l'arrêté pris par le ministre Chaptal. À côté des musées classés se trouvaient également des "musées contrôlés», sur lesquels l'État se réservait aussi un droit de regard.

Le contrôle du ministère de la Culture a progressivement recouvert des thématiques variées: arts décoratifs, histoire, archéologie, et finalement écomusées, musées de société et musées techniques. Cela est dû au fait que, contrairement à la direction des musées de France et au Muséum d'histoire naturelle qui disposaient d'un service technique leur permettant d'exercer leur tutelle sur les musées de province, le musée des Sciences et Techniques n'en était pas doté. Créé en 1793 et doté d'un budget propre en 1907, le Muséum national d'histoire naturelle est aujourd'hui un établissement public à caractère scientifique, culturel et professionnel placé sous la tutelle des ministres chargés de l'Enseignement supérieur, de l'Environnement et de la Recherche ${ }^{57}$. Le décret du 27 avril 1948 y avait institué un service national de muséologie d'histoire naturelle chargé notamment « de coordonner l'action des musées d'histoire naturelle sur l'ensemble du territoire et d'en assurer le contrôle ${ }^{58} »$. En parallèle, la direction des musées de France a tout naturellement étendu son champ de compétence pour satisfaire les demandes des musées locaux qui ne relevaient d'aucun service de l'État ${ }^{59}$. Cet élargissement des compétences a pu être d'ailleurs être perçu comme « une prise de conscience plus nette de l'unité fondamentale de la notion de musée ${ }^{60} »$.

56. D. $n^{\circ}$ 46-712, 26 juil. 1946 fixant la liste des musées classés (JO 28 juillet 1946, p. 6705), complété par D., 28 avr. 1953 (JO 3 mai 1953, p. 4048), D., 21 oct. 1957 (JO 26 octobre 1957, p. 10238), D., 12 juin 1961 (JO 17 juin 1961, p. 5451), D., 18 mars 1969 (JO 25 mars 1969, p. 2964) et D., 8 déc. 1982 (JO 15 décembre 1982, p. 11175).

57. D. $\mathrm{n}^{\circ} 2001-916,3$ oct. 2001 relatif au Muséum national d'histoire naturelle, JO 7 octobre 2001, p. 15803 .

58. Art. 3, D. $\mathrm{n}^{0} 48-734,27$ avr. 1948 relatif à l'organisation du service national de muséologie des sciences naturelles, $J O 28$ avril 1948, p. 4132 (également reproduit dans Jean Chatelain, Droit et administration des musées, Paris, La Documentation française, 1993, p. 180).

59. Question écrite de M. Rodolphe Pesce au Premier ministre et relative à la politique du gouvernement en matière de musées techniques (Assemblée nationale, viie législature, JOAN 21 sept. 1981, p. 2706, réponse publiée dans le JOAN Q 30 nov. 1981, p. 3423).

60. Jean Chatelain, Droit et administration des musées, op. cit., p. 153. 
La loi relative aux musées de France abolit toutes ces distinctions. Il ne reste plus aujourd'hui que des «musées de France », chapeautés depuis 2010 par le service des musées de France.

Il s'agit de faire entrer tous les musées au sein d'un groupe uniforme, régi par les mêmes règles: aussi l'appellation «musée de France» "peut être accordée aux musées appartenant à l'État, à une personne morale de droit public ou à une personne morale de droit privé à but non lucratif ${ }^{61} »$. Elle transcende donc également la qualité du propriétaire du musée.

C'est au travers des procédures d'attribution et de retrait que l'on peut définir l'appellation "musée de France», et mesurer son importance. Elle est en effet attribuée par arrêté du ministre chargé de la Culture après avis du Haut Conseil des musées de France ${ }^{62}$. La collectivité territoriale qui souhaite obtenir l'appellation «musée de France » pour son musée doit présenter un dossier « de l'inventaire des biens affectés aux collections du musée, et précisant l'origine de ces biens, de la décision de l'instance délibérante compétente demandant l'appellation "musée de France", d'un document d'orientation précisant les objectifs scientifiques et culturels du musée ainsi que les conditions et les moyens envisagés pour leur mise en œuvre ${ }^{63}[\ldots]$ ». Il est instruit en premier lieu par la direction régionale des affaires culturelles concernée, puis par le service des musées de France. La Commission scientifique nationale des musées de France est amenée à se prononcer. Celle-ci est présidée, au sein du ministère de la Culture, par le directeur général des patrimoines. Elle comprend des membres de droit (dont le responsable du service des musées de France, les chefs de l'inspection des patrimoines et de la création artistique, et les présidents et directeurs de certains musées nationaux) et des personnalités qualifiées, désignées dans les conditions prévues à l'article D. 451-4 du code du patrimoine. De même, l'avis du Haut Conseil des musées de France ${ }^{64}$ est requis. Il s'agit d'une institution composée d'un député, d’un sénateur, de représentants de l'État, de

61. Art. $1^{\mathrm{er}}, \mathrm{L} . \mathrm{n}^{\mathrm{o}} 2002-5,4$ janv. 2002 relative aux musées de France, précitée, codifié à l'art. L. 441-1 du code du patrimoine.

62. Art. R. 451-3 du code du patrimoine.

63. Art. R. 442-1 du code du patrimoine. Voir aussi la Circ. ministre de la Culture et de la Communication, $\mathrm{n}^{0}$ 2004/014, 6 juil. 2004 relative aux procédures d'attribution de l'appellation «musée de France», de retrait de l'appellation et de transfert de la propriété des collections, BO Culture et Communication, $\mathrm{n}^{\circ}$ 144, p. 30.

64. D. $\mathrm{n}^{\mathrm{o}} 2002-628,25$ avr. 2002, pour l'application de la loi 2002-5 du 4 janv. 2002 relative aux musées de France, $\mathrm{JO}_{2} 8$ avril 2002, p. 7742, codifié aux art. R. 430-1 et suivants du code du patrimoine. 
représentants des collectivités territoriales (celles-ci ont donc une place reconnue en termes de musées), de représentants des professionnels du monde des musées et de "personnalités qualifiées ${ }^{65}$ ».

La décision définitive d'octroi de l'appellation fait ensuite l'objet d'un arrêté du ministre chargé de la Culture (et le cas échéant du ministre concerné) publié au Journal officiel de la République française (JO).

Le code du patrimoine met en avant le caractère volontaire de l'obtention de l'appellation ("attribuée à la demande de la ou des personnes morales propriétaires des collections»), qui est également retirée par le ministre de la Culture, après avis conforme du Haut Conseil des musées de France. Ce retrait intervient « lorsque la conservation et la présentation au public des collections cessent de revêtir un intérêt public $^{66} »$. Il peut également se faire à la demande de la collectivité territoriale responsable du musée concerné, mais dans cette hypothèse, il reste assorti de conditions : l'appellation ne peut être retirée que dans un délai de quatre ans après son attribution et après avis conforme du Haut Conseil des musées de France, notamment si le musée concerné a fait l'objet d'un concours financier de l'État ou d'une collectivité territoriale. De plus, certains garde-fous sont posés, comme dans le cadre de la procédure de déclassement des œuvres des collections des «musées de France ». Ne sont pas concernés par le retrait de l'appellation les biens «ayant fait l'objet d'un transfert de propriété en application des articles L. 451-8 à L. 451-10, ou acquis avec des fonds publics ou après exercice d'un droit de préemption [...] ou à la suite d'une souscription publique ». La collectivité territoriale responsable du musée doit donc transférer la propriété de ces biens à un autre musée de France avant d'obtenir le retrait total de l'appellation.

S'il n'y a donc pas de définition précise d'un "musée de France", les caractéristiques essentielles de l'appellation ressortent de ces dispositions. Le fait qu'elle soit attribuée ou retirée par le ministre de la Culture après avis d'une autorité en partie instituée à cet effet démontre la volonté de créer une sorte de label «qualité » pour les musées. L'appellation se présente comme le reflet de la volonté d'une collectivité territoriale ou d'une personne privée de s'investir dans une démarche

65. Art. R. 430-1 du code du patrimoine.

66. Art. L. 442-3 du code du patrimoine. Dans une espèce du 16 avril 1975, La Comédie de Bourges (req. $n^{\circ}$ 96289) le Conseil d'État a rappelé que l'octroi de l'appellation d'une institution (en l'espèce le titre de Centre dramatique national) ne donne pas de droit acquis à son maintien. Néanmoins, ce retrait doit intervenir dans le cadre d'une procédure respectant le principe du contradictoire. 
qualitative pour son musée, celle-ci étant guidée par la loi : ainsi, l'intérêt public de la collection et l'existence d'objectifs scientifiques et culturels en vue de favoriser le rayonnement du musée sont exigés. Lorsqu'il s'agit de la création d'un nouveau musée, par exemple, les services chargés de l'instruction du dossier de demande de labellisation vérifient la cohérence et le bien-fondé du projet, c'est-à-dire son implantation, son environnement, et sa viabilité, notamment en termes de capacité d'attraction et de rayonnement.

Par ailleurs, la loi protège l'appellation «musée de France » et prévoit des sanctions pénales à l'égard de toute institution qui ferait un usage frauduleux de l'appellation. L'article L. 442-5 du code du patrimoine précise que «le fait, pour le fondateur ou le dirigeant, de droit ou de fait, d'une institution ne bénéficiant pas de l'appellation "musée de France" d'utiliser ou de laisser utiliser cette appellation dans l'intérêt de l'institution est puni d'une amende de 15 ooo euros ». "Les personnes morales peuvent être déclarées responsables pénalement du délit prévu à l'alinéa précédent [...]»

L'appellation "musée de France » ainsi présentée ne peut cependant pas se suffire à elle-même pour se présenter comme un gage de qualité vis-à-vis du public. Aussi se définit-elle également par les obligations qui découlent de son attribution.

\section{Les obligations qui découlent de l'appellation « musée de France »: les questions suscitées par la gestion des collections}

Le principe du contrôle scientifique et technique de l'État sur les musées territoriaux est affirmé par les articles L. 410-2 et L. 442-11 du code du patrimoine et ne vise que les musées ayant obtenu l'appellation «musée de France ». Il se manifeste par l'existence de certains impératifs de gestion, liés aux missions essentielles des musées. Ainsi, aux termes de l'article L. 441-2 du code du patrimoine, les musées de France ont pour missions permanentes de:

1. conserver, restaurer, étudier et enrichir leurs collections ;

2. rendre leurs collections accessibles au public le plus large;

3. concevoir et mettre en œuvre des actions d'éducation et de diffusion visant à assurer l'égal accès de tous à la culture ;

4. contribuer aux progrès de la connaissance et de la recherche ainsi qu'à leur diffusion. 
De ces quatre missions fondamentales on peut avancer, d'une part, que le musée a dorénavant un rôle social et que le public doit être sa préoccupation principale et que, d'autre part, il a une vocation scientifique à développer. On laissera de côté le lien entre le musée et son public pour s'intéresser au régime juridique des collections (2.1) et à leurs modalités de classification (2.2). En effet, la volonté de protéger les collections et d'affirmer le contrôle de l'État sur leur gestion peut parfois aller à l'encontre du principe de libre administration des collectivités territoriales.

\subsection{Le régime juridique des collections}

À l'heure actuelle, le droit public consacre trois régimes juridiques distincts pour les collections des musées de collectivités territoriales, qui ont des conséquences différentes sur leur statut. Ces trois régimes juridiques permettent de mettre en avant la théorie de l'échelle de la domanialité publique, dégagée par Léon Duguit.

En premier lieu, le régime général de la domanialité des collections est posé par l'article L. 2112-1 du code général de la propriété des personnes publiques. Celui-ci rappelle que, «sans préjudice des dispositions applicables en matière de protection des biens culturels, font partie du domaine public mobilier de la personne publique propriétaire les biens présentant un intérêt public du point de vue de l'histoire, de l'art, de l'archéologie, de la science ou de la technique [...]». Le code pose ensuite une liste non exhaustive de biens à vocation exclusivement culturelle ${ }^{67}$. Les collections des musées font partie de cette énumération.

Ces dispositions consacrent la fin d'une longue évolution jurisprudentielle, marquée par une difficulté à consacrer l'existence d'un domaine public mobilier. Pour certains auteurs, le code général de la propriété des personnes publiques consacre l'existence d'un domaine public par nature, distinct du domaine public naturel, et dont la qualification ne découlerait d'aucune affectation. En effet, l'incorporation d'un bien au domaine public se fait en principe sous condition de classement ou d'affectation, c'est-à-dire par le biais d'un acte spécial qui déterminera la destination du bien, et indiquera à quelle catégorie de dépendance domaniale il appartient. Cet acte doit être accompagné d'une affectation de fait, c'est-à-dire que l'affectation prévue doit effectivement être réalisée. De même, en application de la règle du parallélisme des formes, la

67. Art. L. 2112-1 du code général de la propriété des personnes publiques. 
sortie d'un bien mobilier du domaine public implique une décision de déclassement et/ou de désaffectation ${ }^{68}$.

Quant au domaine public mobilier, si le code général de la propriété des personnes publiques consacre l'existence d'un domaine public par nature, cela veut dire que ce qui importe n'est plus l'affectation du meuble mais bien ce qu'il représente en lui-même, c'est-à-dire ses qualités intrinsèques "parce que la fonction de la domanialité publique mobilière est de préserver non la destination du bien, mais le bien en lui-même ${ }^{69}$ ».

Mais ce point de vue ne pourrait-il pas être relativisé, et ne pourrait-on pas avancer que, justement, la qualité intrinsèque des objets mobiliers traduit en elle-même une affectation? On se rapproche alors de la théorie du domaine public mobilier développée par Marcel Waline. Selon lui, appartiennent au domaine public mobilier les biens dont la conservation et la mise à disposition au public relèvent de l'objet même du service public, qui sont affectés à perpétuelle demeure à un édifice ou à un ouvrage faisant partie du domaine public, ou bien si, affectés à un service public, leur disparition peut compromettre la gestion du service public ou y apporter une gêne sérieuse ${ }^{70}$. Cette théorie peut permettre d'avancer que l'affectation des biens au domaine public mobilier découle de leur intérêt d'art et d'histoire et que, par conséquent, le critère de l'affectation n'a pas entièrement disparu des dispositions du code général de la propriété des personnes publiques. C’est ce qui ressort de la jurisprudence consacrant le caractère domanial des biens « objet même du service public», ce critère traduisant alors «le stade suprême de l'affectation au service public ${ }^{71} »$. C'est dans ce sens qu'a raisonné le Conseil d'État, dans une espèce relative à l'autorisation de faire des prises de vues dans le musée des Beaux-Arts de Tours (CE, 29 oct. 2012, EURL Photo Josse c/ commune de Tours). Cette question est essentielle, car elle soulève le problème de la sortie du bien du domaine public. Or la consécration de l'existence d'un domaine public par nature implique que tout déclassement ou toute désaffectation est impossible.

68. CE, 20 juin 1930, Marrot, Recueil Lebon, p. 644 ; CE, 20 janv. 1955, Courvoisier, Recueil Lebon, p. 39 ; CE, 9 nov. 1956, Société des Forges d'Hennebont, Recueil Lebon, p. 667. Voir sur cette question Stéphane Duroy, «La sortie des biens du domaine public: le déclassement», AJDA 1997, p. 819-832.

69. Jean-Gabriel Sorbara, «Le domaine public mobilier au regard du Code général de la propriété des personnes publiques ", AJDA 2007, p. 619.

70. Marcel Waline, note sous CAA Nîmes, 4 décembre 1944, État français c/ Brun, Recueil Dalloz, 1946, p. 28-30.

71. Stéphane Duroy, «Biens meubles culturels et cultuels», RFDA 2007, p. 1158. 
Cette hypothèse trouve son fondement dans le fait qu'il n'existe aucune disposition générale relative à la sortie des biens du domaine public mobilier. En effet, même aujourd'hui, le code général de la propriété des personnes publiques souligne qu' " un bien d'une personne publique mentionnée à l'article L. 1, qui n'est plus affecté à un service public ou à l'usage direct du public, ne fait plus partie du domaine public à compter de l'intervention de l'acte administratif constatant son déclassement ${ }^{72}$ ». Néanmoins, on a du mal à imaginer que ces dispositions puissent convenir aux objets mobiliers. Ainsi, comment ne pas considérer que l'entrée dans le domaine public d'un bien meuble présente un caractère définitif et irrévocable? Cette position est partagée par les professionnels des musées, notamment. Pourtant, d'autres avancent que même en l'absence de dispositions expresses, la sortie des biens mobilier du domaine public reste possible ${ }^{73}$. Possible, mais matériellement difficile à réaliser : comment en effet faire perdre à un objet de collection son intérêt public? Sur ce point, on peut rejoindre la position de M. Jacques Rigaud pour qui les collections muséales sont l'essence même du musée, et ne peuvent être sacrifiées sur l'autel de la « variation du goût ${ }^{74}$ ».

En second lieu, les collections peuvent être protégées au titre de la loi du 30 mars 1887 sur la protection des monuments et objets d'art ayant un intérêt historique ou artistique ${ }^{75}$. Ce texte avait pour ambition de placer les immeubles et le mobilier des personnes publiques sous un régime de protection particulier, en dehors de tout critère de domanialité publique : l'objet était en effet de préserver les œuvres (meubles et immeubles) de la destruction. Ces dispositions ont été renforcées par la loi du 31 décembre 1913 sur les monuments historiques, qui vise à protéger également les monuments privés dignes d'intérêt.

L'application de la règlementation relative aux monuments historiques aux collections des musées découle du fait que, jusqu'en 2002, leur régime juridique était fixé par l'ordonnance du 13 juillet 1945 portant organisation provisoire des musées de beaux-arts ${ }^{76}$. Bien que contenant quelques dispositions relatives au statut des collections, ce texte s'est rapidement avéré insuffisant. La réglementation relative aux monuments historiques avait donc vocation à pallier les « lacunes » de l'ordonnance

72. Art. L. 2141-1 du code général de la propriété des personnes publiques.

73. Gilles Wolkowitsch, Archives, bibliothèques, musées, op. cit., p. 105-114.

74. Ibid., p. 11.

75. L. 30 mars 1887 sur la protection des monuments et objets d'art ayant un intérêt historique ou artistique, $J_{3} 3$ mars 1887, p. 1521.

76. Ord. $n^{\circ} 45-1546,13$ juil. 1945 portant organisation provisoire des musées des beauxarts. 
du 13 juillet 1945 et visait toutes les autres catégories de collections. Le régime de protection institué par les dispositions relatives aux monuments historiques pose une distinction entre les meubles classés, «dont la conservation présente, au point de vue de l'histoire, de l'art, de la science ou de la technique, un intérêt public», et les objets «qui, sans justifier une demande de classement immédiat, présentent [...] un intérêt suffisant pour en rendre désirable la conservation ».

La conséquence majeure du classement ou de l'inscription d'un bien mobilier est que tout projet de modification ou de restauration de ces biens est soumis soit à l'autorisation de l'autorité administrative, soit à notification à cette autorité ${ }^{77}$. Un degré de protection supplémentaire est donc franchi par rapport au droit commun du domaine public, dans la mesure où le déclassement d'un objet mobilier classé monument historique ne dépend pas de l'appréciation de la personne publique propriétaire, mais du ministre chargé de la Culture, à la demande de celle-ci. Néanmoins ces dispositions ne permettent pas d'éviter la circulation et la dispersion des collections. En effet, elles n'ont pas vocation à protéger les ensembles, mais biens les objets individuellement. D'un point de vue purement pratique, cette situation est problématique : pour que les collections des musées soient correctement protégées au titre de la législation sur les monuments historiques, il faudrait que chaque œuvre fasse l'objet d'un classement ou d'une inscription. Outre la charge de travail que cela peut engendrer pour l'administration, une telle situation nuit à la définition générale et à la cohérence de l'institution muséale. Il apparaît en effet que les collections muséales peuvent être constituées d'objets qui, pris dans leur individualité, sont protégés par le régime relatif aux monuments historiques. Comment assurer, alors, la protection des autres œuvres? Sur quelles dispositions protectrices s'appuyer pour empêcher une opération de restauration qui risque de nuire à une œuvre?

Enfin, en troisième lieu, la loi relative aux musées de France consacre une domanialité publique "aménagée». L’article L. 451-5 du code du patrimoine pose le principe selon lequel les collections des musées de France appartenant à une personne publique font partie de son domaine public. Il rappelle le principe de l'imprescriptibilité des collections des musées de France ${ }^{78}$, et surtout souligne que les «biens constituant les

77. Art. L. 622-1, L. 622-20, L. 622-7 et suivants, 622-22 et suivants du code du patrimoine.

78. Art. L. 451-3 du code du patrimoine. Voir, pour une application des principes d'inaliénabilité et d'imprescriptibilité, la décision Cass. crim., 16 juin 1992, pourvoi $\mathrm{n}^{\mathrm{o}}$ 91-86829, Le Gémellion, p. 654. 
collections d'un musée de France appartenant à une personne publique font partie de son domaine public et sont, à ce titre, inaliénables ${ }^{79}$ ". Ainsi, avant l'édiction du code général de la propriété des personnes publiques, la domanialité publique et donc l'inaliénabilité des collections de certains musées se déduisait déjà de leur nature même.

Mais la loi permet le transfert de propriété de collection entre personnes publiques ${ }^{80}$, et les dispositions relatives aux musées de France mettent aussi en place une "domanialité publique aménagée ${ }^{81}$ ", en permettant à la personne publique propriétaire d'un musée de France de « déclasser » certains biens, afin, éventuellement, de les aliéner. Plusieurs garde-fous encadrent néanmoins cette possibilité de déclassement.

Ainsi, les œuvres entrées dans les collections par dons ou legs ou acquises avec l'aide de l'État ne peuvent être déclassées. Outre qu'elles peuvent rassurer les donateurs sur l'avenir de leurs dons ou legs, ces dispositions sont aussi un moyen de légitimer l'intervention de l'État dans le processus d'acquisition d'œuvres. Dans ce sens, elles responsabilisent les collectivités territoriales ou établissements publics qui demandent son soutien. Il y a donc un régime de protection «à deux niveaux » : si certaines œuvres peuvent être déclassées, d'autres seront indéfiniment soumises au régime de protection instauré en 2002.

De plus, lorsque l'objet d'une collection d'un musée de France ne relevant pas de l'État a été déclassé, son propriétaire notifie au ministre chargé de la Culture son intention de vendre l'objet, ainsi que le prix qu'il souhaite obtenir de cette cession. Celui-ci dispose alors de deux mois pour décider d'acquérir le bien ${ }^{82}$. Quant au déclassement en lui-même, la loi du 4 janvier 2002 prévoyait qu'il ne pouvait être opéré sans l'avis conforme de la Commission scientifique nationale des musées de France ${ }^{83}$. Cette procédure a fait l'objet d'une réforme dans le cadre de la loi du 18 mai $2010^{84}$. Elle a été maintenue dans son

79. Art. 11, L. $\mathrm{n}^{\mathrm{o}} 2002-5,4$ janv. 2002 relative aux musées de France, repris à l'art. L. 451-5 du code du patrimoine.

80. Art. L. 451-8 du code du patrimoine. Pour une application de cet article, voir l'arrêté du 23 juin 2008 approuvant le transfert de propriété des collections et attribuant l'appellation «musée de France» en application des art. L. 451-8 et L. 442-1 du code du patrimoine, $J O 2$ juillet 2008, p. 10624.

81. Marie Cornu et Nathalie Mallet-Poujol, Droit, ceuvres d'art et musées, Paris, CNRS, 2006, p. 272.

82. Art. D. 451-22 du code du patrimoine.

83. Art. 16, D. $\mathrm{n}^{\mathrm{0}} 2002-628,25$ avr. 2002, pris pour l'application de la loi $\mathrm{n}^{\mathrm{o}} 2002-5 \mathrm{du}$ 4 janvier 2002 relative aux musées de France.

84. L. $\mathrm{n}^{\mathrm{o}}$ 2010-501, 18 mai 2010, visant à autoriser la restitution par la France des têtes maories à la Nouvelle-Zélande et relative à la gestion des collections, JO 19 mai 2010, p. 9210. 
principe, mais les décisions relatives au déclassement des collections sont dorénavant prises par une Commission scientifique nationale des collections. Celle-ci dispose d'un rôle de conseil aussi bien auprès des personnes publiques que des personnes privées et a pour mission de:

[...] définir des recommandations en matière de déclassement des biens appartenant aux collections [...], et de cession des biens [...]; elle peut également être consultée, par les autorités compétentes pour procéder à de tels déclassements ou cessions, sur toute question qui s'y rapporte; donner son avis conforme sur les décisions de déclassement de biens appartenant aux collections des musées de France et d'œuvres ou objets inscrits sur l'inventaire du Fonds national d'art contemporain et confiés à la garde du Centre national des arts plastiques; donner son avis sur les décisions de déclassement de biens culturels appartenant aux autres collections qui relèvent du domaine public; rendre un avis aux personnes privées gestionnaires de fonds régionaux d'art contemporain, lorsque les collections n'appartiennent pas au domaine public, sur les décisions de cession portant sur les biens qui les constituent ${ }^{85}$.

Ainsi, sans poser d'innovation majeure (car le déclassement est une procédure bien établie en matière de domanialité publique), la loi du 4 janvier 2002 pose une procédure spécifique, plus protectrice que la procédure de droit commun ou que celle relative aux monuments historiques. Un degré de plus est en effet franchi dans la protection puisque la collectivité territoriale propriétaire ne peut déclasser une œuvre elle-même. Elle doit faire appel à une autorité compétente, spécialement instituée à cet effet.

Cette procédure a permis à l'État d'affirmer son pouvoir régalien en la matière. En effet, avant 2002, aucune disposition législative n'avait posé de principe d'inaliénabilité des collections des musées, qu'il s'agisse des musées nationaux ou des musées territoriaux sur lesquels l'État s'était arrogé un droit de regard. Pour autant, une tentative de contrôle avait été initiée dans ce domaine, dans le cadre d'une circulaire des ministres de l'Éducation nationale et de l'Intérieur. Ainsi, une circulaire du 23 avril 1948 rappelait aux préfets les procédures à suivre en matière d'acquisition d'œuvres : à cette époque, l'ordonnance de 1945 requerrait l'avis du ministre de l'Éducation. Appliquant la règle du parallélisme des formes, la circulaire enjoignait les préfets à reproduire cette même

85. Art. 2, L. $\mathrm{n}^{\mathrm{o}}$ 2010-501, 18 mai 2010, visant à autoriser la restitution par la France des têtes maories à la Nouvelle-Zélande et relative à la gestion des collections et codifiée à l'art. L. 115-1 du code du patrimoine. Voir également le D. no 2011-160, 8 févr. 2011 relatif à la commission scientifique nationale des collections, JO 10 février 2011, p. 2588. 
procédure dès lors qu'il était envisagé de faire sortir une œuvre des collections d'un musée classé ou contrôlée ${ }^{86}$. Ces dispositions ne semblent pas avoir eu d'application concrète et sont tombées dans l'oubli, car le projet de loi relatif aux musées de France n'envisageait même pas la question d'un déclassement possible des collections, tant il paraissait évident qu'elles étaient inaliénables. Les mécanismes de déclassement présentés plus haut ont été ajoutés à l'initiative des sénateurs, au nom de la dynamisation des collections et d'un droit de repentir. L'idée ainsi mise en avant était que les collections des musées ne doivent pas être figées dans le temps, et qu'elles doivent pouvoir évoluer afin éventuellement, de se "débarrasser" d'objets sans véritable valeur scientifique, éventuellement en les aliénant. Le produit des aliénations devrait alors permettre l'acquisition de nouvelles œuvres.

Malgré l'émotion suscitée par cette procédure de déclassement dans le milieu des professionnels de musées, elle n'avait jamais été utilisée jusqu'à «l'affaire des têtes maories».

Depuis une vingtaine d'années, le gouvernement néo-zélandais a chargé le musée Te Papa Tongawera de Wellington de s'occuper du rapatriement gratuit des dépouilles des ancêtres du peuple maori, dispersées à travers le monde. Dès le XVIII ${ }^{\mathrm{e}}$ siècle en effet, les colons se sont intéressés à la pratique du tatouage et de la conservation des têtes des hauts dignitaires maoris par leur famille après leur mort. Le commerce instauré alors a tourné à la barbarie, car les maoris se sont mis à tatouer leurs esclaves et à les décapiter une fois leur tête cicatrisée. Depuis 1987, trois cents vingt-deux têtes maories sont revenues en Nouvelle-Zélande (sur les cinq cents recensées) et ont bénéficié d'une inhumation conforme aux rites de la culture maorie. En 2007, le musée de Wellington s'est rapproché de la ville de Rouen, qui conservait une tête maorie dans les réserves de son musée de sciences naturelles depuis 1875. Par une délibération du 19 octobre 2007, le conseil municipal de la ville de Rouen a autorisé la restitution de cette tête maorie et la signature de l'accord formalisant les modalités de restitution entre la ville de Rouen et le musée de Wellington. Cette tête était entrée dans les collections du musée en 1875 , grâce à la donation d'un particulier, procédure qui interdit, comme on l'a vu plus haut, tout déclassement et toute aliénation. La municipalité de Rouen a, par conséquent, décidé de se positionner sur le terrain de la loi bioéthique et de l'article 16-1

86. Circ. Éducation nationale et Intérieur, $\mathrm{n}^{\circ}{ }_{203} \mathrm{AD}_{4}, 23$ avr. 1948, citée par Guillaume Wolkowitsch, Archives, bibliothèques, musées, op. cit., p. 111-114. 
du code civil, qui interdit la patrimonialisation des éléments du corps humain.

$\mathrm{M}^{\text {me }}$ Christine Albanel, alors ministre de la Culture et de la Communication, s'est opposée à cette restitution. Les préfets de la région Haute-Normandie et du département de Seine-Maritime ont donc saisi le juge des référés du tribunal administratif de Rouen afin de suspendre l'exécution de cette délibération. Dans cette affaire, l'ironie est que ce n'est pas la restitution en elle-même qui était reprochée à la commune, mais bien la non-application des dispositions de la loi du 4 janvier 2002 relative aux musées de France. Ainsi l'État a-t-il profité de l'affaire de la tête maorie pour mettre en œuvre pour la première fois son pouvoir de contrôle sur le statut des collections des musées territoriaux dotés de l'appellation «musée de France». Cependant, la question pouvait paraître épineuse car, loin de ne viser qu'un simple « objet», le déclassement concernait des restes humains. Or, leur statut ne fait l'objet d'aucune disposition particulière, hormis les dispositions du code général des collectivités territoriales sur les cimetières ${ }^{87}$, et celles du code pénal relatives à la profanation des cadavres ${ }^{88}$. En principe, les restes humains conservés dans les collections des musées ne devraient pas bénéficier d'un traitement de faveur particulier ${ }^{89}$. Aussi le tribunal administratif de Rouen a-t-il considéré que l'article 16-1 du code civil n'était pas exclusif de toute autre réglementation relative à la domanialité publique ${ }^{90}$.

Selon la cour administrative d'appel, cependant, «les dispositions sus-rappelées du code du patrimoine, qui rendent inaliénables les biens d'une personne publique constituant une collection des musées de France, placent ces biens sous un régime de protection particulière distinct du droit patrimonial énoncé à l'article 16-1 du Code civil ${ }^{91}$ ». Cette argumentation, qui va à l'encontre de toute tentative de restitution, suit les conclusions du commissaire du gouvernement fondées sur une

87. Art. L. 2213-7 et suivants du code général des collectivités territoriales (ci-après "CGCT»).

88. Art. L. 225-17 et suivants du code pénal.

89. Voir l'art. II, 1, E, Circ. Culture et Communication, $\mathrm{n}^{\mathrm{o}}$ 2007/007 portant charte de déontologie des conservateurs du patrimoine (fonction publique d'État et territoriale) et autres responsables scientifiques des musées de France pour l'application de l'art. L. 442-8 du code du patrimoine, BO Culture et Communication, $\mathrm{n}^{\circ}$ 160, p. 15. Voir également Laure Cadot, «Les restes humains : une gageure pour les musées? ", La lettre de l'OCIM, $\mathrm{n}^{\circ}$ 109, 2007, p. 4-15.

90. TA Rouen, 27 déc. 2007, préfet de Seine-Maritime (JCP A 2008, $\mathrm{n}^{\circ}$ 5, p. 27, note Olivier Amiel ; voir aussi JCP G, no 9-10, p. 38, note Colette Saujot).

91. CAA Douai, 24 juil. 2008, Déc. $\mathrm{n}^{\circ}$ 08DA00405, conclusions Jacques Lepers, AJDA 2008, p. 1896. 
conception particulière du patrimoine de l'administration, distinct de la notion de patrimoine développée par la théorie civiliste, elles avancent l'idée que le patrimoine culturel, émanation de la nation, dépasse la notion d'appropriation, et donc de propriété. Dans cette logique, la propriété publique ne serait pas un droit, mais une charge. Elle implique alors l'existence d'un droit de la conservation et exceptionnellement un droit de l'échange. Ce raisonnement exclut alors l'application de l'article 16-1 du code civil, dont l'application se trouve empêchée également par l'aspect scientifique et culturel de la tête maorie.

Cette approche du juge d'appel n'est pas sans rappeler la position du gouvernement à propos de la dépouille de Saartjie Baartman. Cette femme, surnommée «la Vénus hottentote», présentait une morphologie très particulière. Elle avait été vendue en Grande-Bretagne, puis en France, et exhibée comme un phénomène de foire ${ }^{92}$. En 2002, le Parlement a légiféré pour permettre à l'Afrique du Sud de récupérer sa dépouille, alors conservée dans les réserves du Muséum national d'histoire naturelle ${ }^{93}$. En l'espèce, un "simple " déclassement, à la place d'une loi, était possible. La loi a cependant mis fin à la position " attentiste » et hésitante du gouvernement sur cette question. Après hésitations, celui-ci s'est fondé sur le terrain de la loi «bioéthique " pour refuser la restitution. Il a avancé que «le principe d'inaliénabilité n'est pas applicable en raison de la nature même des pièces concernées : s'agissant de restes humains, le squelette ne pourrait, en application de l'article 16-1 du Code civil, faire l'objet d'un droit patrimonial ${ }^{94}$ ». Il ressortait alors de cette argumentation que la dépouille de Saartjie Baartman ne pouvait être considérée comme la propriété de l'État français, ce qui excluait l'emploi des règles relatives à la domanialité publique. Le gouvernement a alors justifié la conservation de ces restes humains au muséum par le fait que "l'inclusion d'une pièce non susceptible de propriété dans une collection publique n'est pas irrégulière, dès lors qu'elle est scientifiquement motivée ${ }^{95} »$.

92. Gérard Badou, L'énigme de la Vénus Hottentote, Paris, Payot, 2002, 188 p.

93. L. $\mathrm{n}^{\mathrm{O}} 2002-323,6$ mars 2002, relative à la restitution par la France de la dépouille mortelle de Saartjie Baartman à l'Afrique du Sud, JO 7 mars 2002, p. 4265.

94. Jean Le Garrec, Rapport sur la proposition de loi, adoptée par le Sénat, relative à la restitution par la France de la dépouille mortelle de Saartjie Baartman à l'Afrique du Sud, Assemblée nationale, $\mathrm{xxi}^{\mathrm{e}}$ législature, $\mathrm{n}^{\mathrm{o}}$ 3563, 2002, p. 11.

95. Philippe Richert, Rapport sur la proposition de loi de Monsieur Nicolas About autorisant la restitution par la France de la dépouille mortelle de Saartjie Baartman, dite "Vénus hottentote " à l'Afrique du Sud, Sénat, rapport $\mathrm{n}^{0} 177$, session ordinaire 2001-2002, p. 8 . 
Partant d'une argumentation différente, la cour administrative de Douai a posé une conclusion similaire. Dans l'affaire de la tête maorie de Rouen il n'y avait pas non plus de droit de propriété mais un droit de garde, qui pouvait exclure la restitution.

S’inspirant de cet antécédent, une proposition de loi visant la restitution des têtes maories conservées dans les collections des musées de France a été déposée au Sénat en $2008^{96}$. Elle a abouti à la loi du 18 mai 2010 visant à autoriser la restitution par la France des têtes maories à la Nouvelle-Zélande et relative à la gestion des collections ${ }^{97}$, dont l'article $1^{\text {er }}$ déclasse officiellement les têtes maories conservées dans les musées de France, afin de faciliter leur restitution. La formulation n'est cependant pas sans ambiguïté : c'est une loi qui «autorise» la restitution des têtes maories, mais son article $1^{\text {er }}$ dispose qu' ' à compter de la date d'entrée en vigueur de la présente loi, les têtes maories conservées par des musées de France cessent de faire partie de leurs collections pour être remises à la Nouvelle-Zélande ». Elle reprend, dans les mêmes termes, la formulation posée par la loi du 6 mars 2002 relative à la restitution de la dépouille de Saartjie Baartman. Outre que le législateur, comme en 2002, applique un vocabulaire propre à exclure l'application des dispositions du code civil, il élude la question de la propriété des collections : il n'est pas en effet question de transfert de propriété ici.

De plus, cette loi démontre la volonté de l'État de se positionner en tant qu'autorité de référence sur la question du statut des collections des musées. En effet, si elle a vocation à « autoriser» la restitution des têtes maories, le texte même indique que les collectivités qui disposent, dans leurs collections, de têtes maories, n'ont aucun pouvoir de décision : toutes les têtes maories conservées dans les collections des musées de France sont déclassés par ces dispositions législatives. Il ne reste plus aux collectivités territoriales détentrices de têtes maories qu'à " prendre acte » du déclassement opéré par le législateur. C'est ce qu'a fait le conseil municipal de la ville de Rouen dans sa délibération du 21 mai $2010^{98}$.

96. Catherine Morin-Desailly, Proposition de loi visant à autoriser la restitution par la France des têtes maories, Sénat, rapport $\mathrm{n}^{0} 215$, session ordinaire 2007-2008.

97. L. $\mathrm{n}^{\mathrm{o}}$ 2010-501, 18 mai 2010, visant à autoriser la restitution par la France des têtes maories à la Nouvelle-Zélande et relative à la gestion des collections, JO 19 mai 2010, p. 9210.

98. Délibération du conseil municipal de la ville de Rouen (10-7), 21 mai 2010 relative à la restitution d'une tête maorie à la Nouvelle-Zélande, disponible en ligne à l'adresse suivante: http://www.rouen.fr/deliberation/dl20100521 \_10 \_7 (consultée le 5 novembre 2015). 
Au-delà de la question des têtes maories, la loi du 18 mai 2010 pose le principe de la réforme de la procédure de déclassement des collections. Outre qu'elle vise ainsi les œuvres et objets susceptibles de revendications (et dans ce cas, il aurait peut-être fallu encadrer plus les conditions de restitution - «le pays d'origine d'un peuple contemporain [devait avoir] formulé la demande de restitution de ce bien; que celui-ci ne fasse pas l'objet de recherches scientifiques; qu'il ne soit pas destiné à être exposé ni conservé dans des réserves dans le pays d'origine mais qu'il soit inhumé ; qu'il soit issu d'actes de barbarie ayant entraîné la mort ${ }^{99}$ »pour éviter des revendications inconsidérées), elle s’inscrit dans un débat plus profond, celui de la question de l'inaliénabilité des collections. Audelà du caractère politique et symbolique des restitutions, se pose en effet la question du déclassement des collections des musées de France dans un but purement économique. Je n'ai malheureusement pas de temps à consacrer à cette question, mais il faut bien noter que l'affaire de la tête maorie de Rouen est apparue dans un contexte tendu, lié à la question de déclassement et d'aliénabilité des collections ${ }^{100}$.

\subsection{Les méthodes de classification des collections: l'inventaire et le récolement des collections}

L'article 12 de la loi "musées » codifié à l'article L. 451-2 du code du patrimoine dispose que «les collections des musées de France font l'objet d'une inscription sur un inventaire». "Il est procédé à leur récolement tous les dix ans. »Ces dispositions posent un fondement législatif aux méthodes de classification des collections des musées de France, qui n'est pas sans susciter des interrogations, liées au statut des collections et aux modalités de l'exercice du contrôle de l'État sur les musées de France en la matière.

Légalisé en 2002, l'inventaire est un «document administratif, justifiant à un moment donné la présence, l'état des collections et leur

99. Catherine Morin-Desailly, Proposition de loi visant à autoriser la restitution par la France des têtes maories, op. cit., p. 3.

100. Maurice Lévy et Jean-Pierre Jouyet, L'économie de l'immatériel, la croissance de demain, ministère de l'Économie, des Finances et de l'Industrie, 2006, p. 105-107; Jean-François Mancel, Proposition de loi tendant à établir une réelle liberté de gestion des établissements culturels, Assemblée nationale, xii ${ }^{e}$ législature, rapport $\mathrm{n}^{\mathrm{o}} 233,2007$; Jacques Rigaud, Réflexions sur les possibilités pour les opérateurs publics d'aliéner des cuvres de leurs collections, rapport au ministre de la Culture et de la Communication, 2008 ; Françoise Benhamou et David Thesmar, Valoriser le patrimoine culturel de la France, Conseil d'analyse économique, 2011. 
provenance ${ }^{101}$ ». Il constitue alors à la fois la fiche d'identité d'une œuvre et un acte légal qui justifie et institutionnalise l'entrée d'un bien dans une collection, et donc sa propriété. Mené dans les conditions posées par les actes réglementaires d'application de la loi relative aux musées de France ${ }^{102}$, il vise « tout bien acquis à titre gratuit ou onéreux affecté aux collections du musée de France par un acte émanant de la personne morale propriétaire du bien ${ }^{103}$ ", et doit en principe être doublé d'un enregistrement comptable au titre des immobilisations de la collectivité territoriale gestionnaire du musée ${ }^{104}$. Mais si l'inventaire est un élément indispensable à la bonne administration des collections, il n'est pas suffisant: une fois réalisé, il importe de vérifier que ce qui y est inscrit est toujours valable. Cette opération s'appelle le récolement, et consiste "à vérifier, sur pièce et sur place, à partir d'un bien et de son numéro d'inventaire, la présence du bien dans les collections; sa localisation ; l'état du bien; son marquage ; la conformité de l'inscription à l'inventaire avec le bien ainsi que, le cas échéant, avec les différentes sources documentaires, archives, dossiers d'œuvres, catalogues ${ }^{105}$ ».

Ainsi, on peut se demander si l'inscription d'un bien à l'inventaire d'un musée est une condition essentielle à son entrée dans la collection "musée de France» et à sa soumission au régime juridique qui en découle. Les avis des professionnels divergent en la matière. Mais, la formulation de l'arrêté du 25 mai 2004, qui dispose que «l'inscription à l'inventaire d'un bien affecté aux collections d'un musée de France intervient au plus tard le 31 décembre de l'année suivant l'année de l'acquisition ", suggère que c'est l'acquisition qui traduit l'affectation, et non le report sur la liste des collections du musée. La doctrine

101. Gérard Guillot-Chêne, dans Marie-Odile de Bary et Jean-Michel Tobelem (dir.), Manuel de muséographie, petit guide à l'usage des responsables de musées, Biarritz, Option culture, 1998, p. 181.

102. D. $\mathrm{n}^{\circ} 2002-852,2$ mai 2002, pris en application de la loi $\mathrm{n}^{\circ} 2002-5$ du 4 janvier 2002 relative aux musées de France, précité et codifié, pour ses dispositions relatives à l'inventaire, aux art. D. 451-15 et suivants du code du patrimoine; A. min. 25 mai 2004, fixant les normes techniques relatives à la tenue de l'inventaire, du registre des biens déposé dans un musée de France et au récolement, JO 12 juin 2004, p. 10483 .

103. Art. D. 451-18 du code du patrimoine.

104. Voir Chambre régionale des comptes de Franche-Comté dans son Rapport d'observations définitives sur la gestion de la commune d'Ornans de 2007, p. 12 (à propos des collections du musée Gustave-Courbet).

105. Art. 11, A. 24 mai 2004, fixant les normes techniques relatives à la tenue de l'inventaire, du registre des biens déposé dans un musée de France et au récolement. 
s'accorde également sur ce principe, et le juge administratif s'est rallié à cette interprétation, qui peut traduire l'existence d'une domanialité par nature: dans l'affaire de la tête maorie de Rouen, le tribunal administratif de Rouen a jugé que la non-inscription de la tête maorie sur l'inventaire du musée n'était pas une condition suffisante pour déroger à la procédure de déclassement prévue par la loi relative aux musées de France ${ }^{106}$. Le juge administratif a sans doute pris la mesure des lacunes constatées en matière d'inventaire dans les musées nationaux comme dans les musées territoriaux. Car en principe, l'inventaire est prospectif (chaque objet qui entre au musée est inventorié). Mais l'obligation d'inventorier étant récente, de nombreux biens ne sont toujours pas inscrits sur le registre d'inventaire: pendant longtemps en effet la direction des musées de France ne s'est pas intéressée à la question de l'inventaire, entraînant la généralisation de pratiques particulières et propres à chaque établissement. Mais si la constitution d'un inventaire a fini par devenir une nécessité, pour les musées de France ${ }^{107}$, de nombreuses lacunes ont été soulevées dans certaines institutions. Le musée Carnavalet de la ville de Paris, par exemple, est constitué d'un fonds de plus de 400 ooo œuvres, réparties dans des collections d'objets archéologiques, de mobilier, de numismatique, de dessins, d'objets d'art, de peintures, de sculptures et estampes, dont le nombre s'élève approximativement à 230000 . L'inventaire, en plus d'être prospectif, se doit d'être rétrospectif.

Dès lors, la position du ministère de la Culture et du juge administratif sur la question de l'inventaire permet de protéger l'ensemble des œuvres des collections des musées de France. Mais, dans ce cas, quel régime doit-on appliquer aux objets qui ne rentrent pas dans la catégorie des collections «musées de France »? En effet, l'acquisition d'une œuvre par un musée doté de l'appellation "musée de France » est subordonnée à l'avis d'une commission scientifique ad hoc ${ }^{108}$. Le principe de libre administration des collectivités territoriales implique que celles-ci ne sont pas liées par cet avis. Il peut donc arriver qu'une collectivité territoriale acquière une œuvre pour son musée, et qu'elle se voit

106. TA Rouen, 27 déc. 2007, Préfet de Seine-Maritime.

107. Dans une décision du 19 mai 1989, le Conseil d'État a considéré qu'en «méconnaissant gravement les règles de la comptabilité publique et en négligeant, malgré les observations qui lui avaient été faites, de tenir les livres d'inventaire des collections du musée, [le conservateur du musée des beaux-arts de la ville de Chartres] a commis des fautes d'une gravité » justifiant sa révocation (CE, 19 mai 1989, Ville de Chartres, Recueil Lebon, Tables, p. 467 et 757).

108. Art. L. 451-1 et L. 452-1 et suivants du code du patrimoine. 
refuser l'application du régime "musée de France». Dans ce cas, le statut des collections peuvent varier, au sein même du musée. On aura alors affaire à trois types de régimes juridiques, qui impliquent que les œuvres ne puissent être traitées de la même manière: le régime juridique de droit commun, qui découle de l'application des dispositions de l'article L. 2112-1 du code général de la propriété des personnes publiques; le régime relatif aux monuments historiques et le régime relatif aux collections des musées de France.

Comment alors faire la distinction entre les œuvres dotées de l'appellation «musées de France» et non encore inventoriées et les autres? Faudrait-il alors recourir à un inventaire séparé? D'un point de vue juridique, cette solution ne semble pas pertinente. Elle risque en effet d'entraîner une dispersion dans les documents administratifs relatifs à la gestion des collections, d'autant que, au regard des dispositions réglementaires portant application de la loi relative aux musées de France, il ne peut exister que deux inventaires: l'un pour les collections du musée, l'autre pour les dépôts.

L'inventaire, comme le récolement, doivent être effectués par une personne compétente d'un point de vue scientifique, et donc, en principe un conservateur ${ }^{109}$. Sont ici visés principalement les conservateurs $\mathrm{du}$ patrimoine (conservateurs territoriaux du patrimoine), dont les modalités de recrutement traduisent également une volonté de contrôle de l'État sur les musées territoriaux, difficile parfois, à mettre en œuvre dans le cadre du principe de libre administration des collectivités territoriales.

Le terme même de «conservateur » souligne l'essence du musée. La profession de conservateur s'est imposée durant l'entre-deux-guerres face aux artistes, érudits, amateurs et collectionneurs qui incarnaient parfois la mémoire locale à la tête des musées. Sa légitimation est pleinement liée à l'affirmation et au développement des préceptes liés à la muséographie.

La question de la professionnalisation des musées s'est posée cependant dès la Révolution française. Le Comité d'instruction publique a ainsi adopté, le 15 mars 1794, une Instruction sur la manière d'inventorier et de conserver tous les objets qui peuvent servir aux arts, aux sciences et à l'enseignement. Des principes de gestion des collections ont également été définis sous la $\mathrm{II}^{\mathrm{e}}$ République et en 1874 , un décret portant

109. Art. L. 442-8 du code du patrimoine. 
organisation des musées nationaux a précisé et formalisé les missions du personnel des musées de l'État ${ }^{110}$.

En 1882, Antonin Proust, ministre des Arts ${ }^{111}$, a créé l'École du Louvre, qui avait pour vocation première de former les conservateurs et autres professionnels du monde des musées. Parcours incontournable pour tout conservateur encore aujourd'hui, elle dispensait un enseignement qui permettait aux élèves diplômés de devenir conservateur de musée. Leur recrutement passait par une inscription sur une liste d'aptitude dressée par une commission de classement des personnels scientifiques (article 5 de l'ordonnance de 1945 portant organisation provisoire des musées de beaux-arts). Les premiers concours pour le recrutement des conservateurs des musées nationaux ont été organisés en 1963, en application du nouveau statut des fonctionnaires fixé par l'ordonnance du 4 février $1959^{112}$ et des dispositions propres au statut des conservateurs ${ }^{113}$.

Dès son origine, l'École du Louvre avait également pour mission de former les conservateurs des musées territoriaux. En effet, bien avant l'institutionnalisation du système de recrutement par concours, voire de la création de l'École du Louvre, le pouvoir central a cherché à contrôler le recrutement des responsables de musées territoriaux. L'argument principal en faveur de l'intervention de l'État sur les musées territoriaux était fondé sur sa volonté de contrôler la bonne conservation des dépôts qu'il avait effectués dans les musées tout au long du siècle. Le recrutement des conservateurs de musées territoriaux par le préfet a pu conduire à une forme d'instrumentalisation politique du musée et l'État a encouragé à plusieurs reprises les préfets à satisfaire les demandes des communes en fonction de la couleur politique des élus locaux ${ }^{114}$, ou à refuser de valider les clauses liées au salaire des responsables de musées qui n'étaient pas conservateurs d'État dans le budget des communes. Par la suite, et avec la création de l'École du Louvre, les conservateurs des

110. D., 6 mars 1974 complété par D., $1^{\text {er }}$ mars 1879. Voir Frédéric Poulard, Conservateurs de musées et politiques culturelles, op. cit., p. 19.

111. Homme politique, journaliste, critique d'art et ami du peintre Manet, Antonin Proust (1832-1905) est considéré comme le tout premier ministre de la Culture en France. Sous la III $^{\mathrm{e}}$ République, Léon Gambetta, président du conseil, a fait appel à lui pour la constitution de son "grand ministère». Antonin Proust est devenu ministre des Beaux-Arts du 14 novembre 1881 au 26 janvier 1882.

112. Ord. $\mathrm{n}^{0}$ 59-244, 4 févr. 1959 relative au statut général des fonctionnaires, JO 8 février 1959, p. 1747 (art. 18).

113. D. $\mathrm{n}^{\circ} 63-973,17$ sept. 1963 relatif au statut particulier des membres de la conservation des musées de France, JO 23 septembre 1963, p. 8620.

114. Chantal Georgel, «L'État et "ses" musées de province... », art. cité, p. 65. 
musées classés et contrôlés devaient être diplômés de l'École du Louvre et inscrits sur une liste d'aptitude, de la même manière que pour les conservateurs des musées nationaux.

Le principe de l'inscription sur une liste d'aptitude a perduré après l'institution du principe du concours en 1963 , mais il ne concernait plus que le recrutement des conservateurs des musées contrôlés. Les conservateurs des musées classés étaient donc, finalement, des conservateurs d'État recrutés sur concours ${ }^{115}$. Ils se retrouvaient dans une situation assez particulière : recrutés par le ministre chargé de la Culture, ils étaient considérés comme des agents relevant de la collectivité territoriale propriétaire du musée, et rémunérés par elle ${ }^{116}$.

Ce mode de recrutement a pris fin en 1986, date à laquelle a aussi été créée une institution spécialisée chargée de leur formation: l'École du patrimoine. Rattachée à l'École du Louvre, cette institution avait pour mission de prendre en charge le recrutement par concours et la formation de tous les conservateurs, y compris ceux des musées contrôlés. Il a néanmoins fallu attendre 1992 pour que les conservateurs territoriaux soient dotés d'un statut propre ${ }^{117}$, en grande partie calqué sur statut du corps des conservateurs du patrimoine relevant de la fonction publique d'État ${ }^{118}$.

Une collectivité territoriale peut recruter, pour assurer les activités scientifiques de son musée, un conservateur du patrimoine, mais également un attaché de conservation du patrimoine ${ }^{119}$, un assistant de conservation du patrimoine ${ }^{120}$ ou un bibliothécaire ${ }^{121}$. Elle peut aussi recourir aux agents de l'État (chargés d'études documentaires,

115. Ancien art. 10 de l'ordonnance du 13 juillet 1945 portant organisation provisoire des musées de beaux-arts, rappelé à l'art. 62 de la loi n ${ }^{\circ} 83-663$ du 22 juillet 1983 complétant la loi $\mathrm{n}^{\mathrm{O}} 83-8 \mathrm{du} 7$ janvier 1983 relative à la répartition des compétences entre les communes, les départements, les régions et l'État, JO 23 juillet 1983, p. 2286.

116. CE, 16 juil. 1976, Ville de Dunkerque, Recueil Lebon, p. 409.

117. D. $\mathrm{n}^{\circ}$ 91-839, 2 sept. 1991 portant statut particulier du cadre d'emplois des conservateurs territoriaux du patrimoine, JO 4 septembre 1991, p. 11572.

118. D. $\mathrm{n}^{\circ}$ 90-404, 16 mai 1990, portant statut particulier du corps des conservateurs du patrimoine, JO 17 mai 1990, p. 5904; D. n 90-405, 16 mai 1990 portant statut particulier des conservateurs généraux du patrimoine, JO 17 mai 1990, p. 5909.

119. D. $\mathrm{n}^{\circ}$ 91-843, 2 sept. 1991 portant statut particulier du cadre d'emplois des attachés territoriaux de conservation du patrimoine, JO 4 septembre 1991, p. 11584.

120. D. $\mathrm{n}^{\circ}$ 95-33, 10 janv. 1995 portant statut particulier du cadre d'emplois des assistants territoriaux de conservation du patrimoine et des bibliothèques, JO 12 janvier 1995, p. 579.

121. D. $\mathrm{n}^{\circ} 91-845,2$ sept. 1991 portant statut particulier du cadre d'emplois des bibliothécaires territoriaux, JO 4 sept. 1991, p. 11589. 
secrétaires de documentation) par la voie du détachement. Les agents territoriaux sont en principe recrutés dans le respect des règles générales relatives à la fonction publique territoriale, c'est-à-dire par concours avec inscription des lauréats sur une liste d'aptitude pendant trois ans. Il en est de même pour les conservateurs, qui suivent cependant toujours la formation dispensée par l'École du patrimoine, devenu Institut national du patrimoine en $2001^{122}$. Il organise le concours et la formation de tous les conservateurs du patrimoine (spécialité musée bien sûr, mais aussi archives, archéologie, bibliothèque, inventaire et monuments historiques), quelle que soit la fonction publique à laquelle ils sont rattachés: la spécificité du métier de conservateur est telle que le Centre national de la fonction publique et la ville de Paris, qui dispose pourtant d'une fonction publique propre, passent des conventions avec cette institution. Les conservateurs sont ainsi tous recrutés et formés de la même façon.

Le contrôle scientifique et technique de l'État est donc large puisque, au-delà de l'obligation de recourir à des fonctionnaires appartenant à des cadres d'emplois ayant vocation à exercer des missions scientifiques, c'est une institution de l'État qui forme ces fonctionnaires.

Si l'État s'est chargé, dès la fin du XIx ${ }^{\mathrm{e}}$ siècle, de former les conservateurs des musées territoriaux, les collectivités territoriales peuvent cependant « contourner » cette obligation en recrutant, pour la gestion de leurs collections, des attachés de conservation du patrimoine ${ }^{123}$.

La création de ce cadre d'emplois a suscité quelques interrogations, car l'attaché de conservation du patrimoine est doté de missions et de prérogatives similaires à celles du conservateur. Les conditions de son recrutement sont cependant nettement différentes: les attachés territoriaux de conservation sont recrutés dans le cadre de concours organisés par les centres de gestion de la fonction publique territoriale. Ils ne suivent pas la formation de l'Institut national du patrimoine prévue pour les conservateurs. En principe, ils peuvent être nommés à des postes de direction, mais dans les musées dotés d'un conservateur, ils ont vocation à remplir la fonction d'adjoint du conservateur. Il s'agit donc d'un « corps "intermédiaire" permettant de densifier les équipes de conservation par la présence de collaborateurs du ou des conservateurs ou de se substituer au conservateur lui-même lorsque le poste ne légitimait pas sa

122. Art. 2, D. n ${ }^{\circ} 90-406,16$ mai 1990 créant et organisant l'École nationale du patrimoine, précité, modifié le 22 décembre 2001.

123. Art. 2, D. $\mathrm{n}^{\circ} 91-843,2$ sept. 1991 portant statut particulier du cadre d'emplois des attachés territoriaux de conservation du patrimoine, JO 4 septembre 1991, p. 11584 . 
présence $^{124} »$. Sans remettre en cause la qualité des prestations fournies, Le livre blanc des musées de France, publié en 2011 par l'association générale des conservateurs des collections publiques de France, note un recours croissant aux attachés territoriaux de conservation. Ceux-ci ayant un grade inférieur $(A)$ à celui des conservateurs $(A+)$, le niveau de rémunération peut alors être l'argument des collectivités; cela aurait à terme pour conséquence de dévaloriser la fonction de directeur de musée ou de responsable scientifique des collections. "Pourquoi irais-je recruter un conservateur quand je peux en faire l'économie et recruter un agent dont la grille indiciaire est moins élevée? »On peut citer ainsi plusieurs cas de remplacements de conservateurs en chef par des attachés. Or, il n'est pas normal d'exiger le même travail et les mêmes responsabilités des agents de statut et donc de rémunération distincts.

Par ailleurs, la loi autorise le recrutement, sur des postes de conservateurs, de personnel non titulaires: l'article 3 de la loi du 26 janvier 1984 rappelle que « des emplois permanents peuvent être occupés par des agents contractuels dans les cas suivants : $1^{\circ}$ Lorsqu'il n'existe pas de cadre d'emplois de fonctionnaires susceptibles d'assurer les fonctions correspondantes; $2^{\circ}$ Pour les emplois du niveau de la catégorie $\mathrm{A}$, lorsque la nature des fonctions ou les besoins des services le justifient ». Cette dernière disposition est régulièrement utilisée par les collectivités territoriales pour justifier le recours à des agents non titulaires. Ceux-ci sont alors recrutés sur la base d'un contrat à durée déterminée de trois ans renouvelables. La durée des contrats successifs ne peut dépasser six ans. Si le contrat est reconduit à l'issue de ces six ans, il prend la forme d'un contrat à durée indéterminée. Il revient alors aux collectivités territoriales de fournir au représentant de l'État dans le département toutes les pièces justifiant, notamment, le recrutement d'un agent non titulaire ${ }^{125}$.

En principe, ce personnel doit disposer de qualifications et d'aptitudes à exercer des compétences scientifiques qui font l'objet d'une reconnaissance particulière. L'article R. 442-5 du code du patrimoine précise ainsi que «[...] présentent les qualifications requises pour exercer la responsabilité des activités scientifiques d'un musée de France dont les collections appartiennent à une personne publique : [...] selon la nature

124. Christophe Vital, Le livre blanc des musées de France, Musées et collections publiques de France, $\mathrm{n}^{\mathrm{o}} 260$, 2010, p. 66.

125. Pour une application de ces dispositions à un musée local, voir CAA Lyon, 8 févr. 1999, Ville de Montluçon, req. nº 96LYo1580. 
des fonctions ou les besoins des services d'un musée de France, les personnes ou catégories de personnes reconnues par un arrêté du ministre chargé de la culture et, le cas échéant, du ministre dont relève le musée en cause ou qui en assure la tutelle comme présentant des qualifications équivalentes à celles des fonctionnaires mentionnés au $1^{\circ}$; cet arrêté est pris après avis d'une commission nationale d'évaluation ${ }^{126}$ ". Cette commission ${ }^{127}$ a vocation à se prononcer sur les qualifications requises pour exercer des activités scientifiques dans un musée de France, pour un poste défini. Cela implique qu'à chaque changement de poste, un nouvel avis de la commission est nécessaire. Elle «donne son avis motivé sur le strict plan scientifique et professionnel, afin de contribuer à rendre aussi élevée et homogène que possible la qualité des recrutements des personnels dans les musées concernés tout en tenant compte de la diversité des domaines de conservation et de mise en valeur du patrimoine muséographique $^{128} »$.

Ce système permet ainsi de respecter la diversité des profils des candidats à l'exercice de missions scientifiques dans un musée. Il reste néanmoins considéré comme une entrée parallèle dans les fonctions de conservateur et, parfois, comme une solution de facilité vis-à-vis du concours. Il est cependant préférable au recrutement de non titulaires dont les qualifications n'ont fait l'objet d'aucune reconnaissance, ce qui semble fréquent dans les musées de France. La question n'est pas de remettre en cause les compétences de ces agents, ni de se laisser aller au corporatisme et au conservatisme dans ce domaine. Il peut cependant paraître étrange, inéquitable en tous cas, de voir que des tâches qui, en principe, sont confiées à des personnes dont les compétences ont été sanctionnées par la réussite d'un concours relativement difficile, puissent être exercées par des personnes qui ne sont pas titulaires de ce concours. De plus, dans de telles conditions, l'État perd l'une de ses prérogatives (la formation du conservateur) et son contrôle sur les musées territoriaux s'en trouve allégé.

De même, la position de l'État par rapport aux obligations d'inventaire et de récolement des collections n'est pas clairement affirmée. Les dispositions législatives et réglementaires précisent que ces missions sont

126. Rédaction issue de l'article 10 du décret 2002-628 du 25 avril 2002 pris pour l'application de la loi $\mathrm{n}^{0}$ 2002-5 du 4 janvier 2002 relative aux musées de France, JO 28 avril 2002, p. 742.

127. Art. R. 442-7 et suivants du code du patrimoine.

128. Circ. Éducation nationale, Enseignement supérieur et Recherche, $n^{0}$ 2004-091, 9 juin 2004 et relative aux qualifications requises de certains personnels des musées de France. 
menées sous la responsabilité des conservateurs qui doivent, lorsqu'ils quittent leurs fonctions, « remettre à la personne morale propriétaire du musée de France un état récapitulatif des biens inscrits sur ces registres qui, après récolement, sont considérés comme manquants ». Quant au récolement, il doit être "mené par campagnes planifiées en fonction de l'organisation du musée, notamment par lieu, par technique, par corpus ou par campagne annuelle ${ }^{129}$ ". Il a été décidé que ce récolement décennal devrait être achevé dans les dix ans qui suivent la date de publication de l'arrêté du 25 mai 2004, soit en $2014^{130}$. Mais si de nombreuses lacunes ont été relevées en matière d'inventaire, on peut imaginer que celles qui concernent le récolement sont plus importantes encore. Les services de l'État sont conscients de la difficulté de certaines institutions (et notamment les musées territoriaux) à mener à bien les opérations d'inventaire, et qui plus est, de récolement, dans les temps impartis par la loi. Mais si la tâche à accomplir paraît difficile, il a également été relevé, outre un retard conséquent dans la parution des décrets d'application de la loi relative aux musées de France, une forme de réticence de la part des professionnels des musées à mettre à exécution les directives législatives ${ }^{131}$. La Commission des affaires culturelles et de l'éducation de l'Assemblée nationale qui a fait ces constats a néanmoins proposé, dans un rapport publié à la fin de l'année 2014, de reporter la date butoir d'achèvement du récolement au 31 décembre 2016.

Dès lors, une vraie question se pose : comment concilier impératifs législatifs et principe de libre administration des collectivités territoriales? S’il revient au préfet, après avis de la direction régionale des affaires culturelles, de rappeler leurs obligations aux collectivités territoriales, ni le législateur, ni le gouvernement ne se sont positionnés sur la manière de sanctionner le non-respect des dispositions de la loi relative aux musées de France par les collectivités territoriales. Dans une décision du 11 octobre 2011, la cour administrative d'appel de Marseille a eu l'occasion d'apprécier la légalité d'une mesure de suspension, puis de mise à la retraite d'office d'un conservateur de musée fondée notamment sur l'abstention de ce dernier à procéder aux opérations de récolement

129. Art. 12, A. 25 mai 2004 fixant les normes techniques relatives à la tenue de l'inventaire, du registre des biens déposé dans un musée de France et au récolement.

130. Circ. 28 juil. 2006, $\mathrm{n}^{0}$ 2006-006 relative aux opérations de récolement des musées de France.

131. Isabelle Attard, Michel Herbillon, Michel Piron et Marcel Rogemont, Rapport sur la gestion des réserves et des dépôts des musées, Commission des affaires culturelles et de l'éducation, Assemblée nationale, xiv ${ }^{e}$ législature, rapport $n^{\circ} 2474$, 17 décembre 2014, p. 31-33. 
des œuvres du musée. En l'espèce, la cour administrative d'appel a considéré que le conservateur ne s'était pas abstenu de procéder au récolement de la collection du musée, et que celui-ci avait bien été entamé. Elle a rappelé que les dispositions réglementaires imposaient que le premier récolement décennal soit achevé en 2014 et que, par conséquent, il ne pouvait être reproché au conservateur de ne pas l'avoir achevé dans les délais impartis par le maire ${ }^{132}$. Cette décision semble impliquer qu'aucune "sanction " ne pouvait intervenir avant 2014, tant en ce qui concerne le récolement, qu'en ce qui concerne l'inventaire des collections. En effet, l'article 2 de l'arrêté du 25 mai 2004 fixant les normes techniques relatives à la tenue de l'inventaire, du registre des biens déposés dans un musée de France et au récolement prévoit que « les biens entrés dans les collections antérieurement à la publication du présent arrêté et non encore inventoriés à cette date sont inventoriés dans le registre d'inventaire au plus tard lors de la première campagne de récolement », soit en 2014. Pour autant, le code du patrimoine prévoit que la responsabilité du conservateur est engagée dans ce domaine. Comment interpréter ce principe, dès lors qu'aucune disposition n'est venue le clarifier?

Alors que les collectivités territoriales se posent comme les premiers promoteurs de musées en France, elles restent soumises au contrôle de l'État, et cela en application du principe constitutionnel de libre administration. L'État intervient de différentes façons dans ce domaine : conseiller, incitateur, prescripteur, mais aussi contrôleur, voire policier. Sa position s'est affirmée avec la mise en œuvre de la loi relative aux musées de France. Il ressort en effet de ses dispositions que l'État a voulu affirmer et légitimer son contrôle sur les musées territoriaux. Cependant, son intervention reste limitée à un contrôle strict de légalité (comme

132. CAA Marseille, 11 oct. 2011, Trotry de la Touche c/ Commune de Menton, req. no $09 \mathrm{MA00612}$ : «[considérant] qu'il est reproché à titre principal à l’intéressé, non de n'avoir pas débuté les opérations de récolement ou de les avoir négligées, mais de ne pas les avoir terminées dans un délai bref imposé par le maire de Menton [...]; qu'aux termes toutefois de l'article 12 de l'arrêté précité qui est entré en vigueur en 2004: le récolement, obligatoire au moins une fois tous les dix ans, est mené par campagnes planifiées en fonction de l'organisation du musée, notamment par lieu, par technique, par corpus ou par campagne annuelle; qu'il résulte de ces dispositions que le terme légal d'achèvement des opérations du premier récolement dont l'intéressé avait la charge était fixé à l'année 2014 seulement [...] ; que dans ces conditions, la conduite par l'appelant des opérations de récolement dont il avait la charge ne révèle aucun fait établi qualifiable de faute disciplinaire et par suite de nature à justifier une sanction. » 
dans l'affaire de la tête maorie) ou du respect de l'intégrité des collections. Ainsi, son «ingérence » est-elle cantonnée à l'hypothèse dans laquelle les collections se retrouveraient en péril au sens de l'article L. 452-2 du code du patrimoine ${ }^{133}$.

La question du suivi de la gestion des collections des musées de France, et du respect des dispositions législatives et réglementaires en la matière peut donc se poser. Outre que cela nécessite, pour le service des musées de France, de disposer de moyens qu'il n'a pas (on a en effet vu précédemment qu'il n'y a pas un conseiller des musées par direction régionale des affaires culturelles), il paraît pertinent de se demander comment sanctionner le non-respect des dispositions relatives à l'inventaire et au récolement des collections. Or, si le nonrespect des impératifs d'inventaire et de récolement était sanctionné par le retrait de l'appellation «musées de France », l'État, qui souhaitait avant tout placer les collections des musées sous un régime protecteur, se trouverait dans une impasse.

En effet, au moment de l'adoption de la loi relative aux musées de France, il était question de vérifier, pour chaque musée classé ou contrôlé, sa capacité à obtenir ou non l'appellation. L'autorité administrative a finalement procédé à une "généralisation » de l'attribution de l'appellation, sans véritablement prendre en compte le souhait des collectivités territoriales en la matière, ni l'aptitude de certains musées à remplir les obligations posées par la loi. Le ministère de la Culture a justifié ce procédé par la nécessité d'assurer une protection juridique des collections des musées. Pourtant, le «toilettage» annoncé en premier lieu «laissait entrevoir la possibilité de recentrer l'effort de l'État sur les établissements répondant à des exigences en matière de présentation et de conservation des œuvres ${ }^{134}$ ». De ce fait, le retrait «sanction» de l'appellation «musée de France» mettrait en avant l'incohérence de la politique de l'État en la matière, et les collections retourneraient dans le domaine public de la collectivité territoriale concernée. Bien sûr, l'État peut veiller à ne pas user de ses prérogatives pour soutenir un musée non respectueux de ses obligations. Mais une telle attitude ne porterait aucun bénéfice aux collections concernées...

133. Art. L. 452-2 du code du patrimoine. Pour une mise en œuvre de ces dispositions, voir TA Pau, 15 déc. 2009, Association régionaliste du Béarn, du pays Basque et des contrées de l'Adour, req. $\mathrm{n}^{0} 0701934$.

134. Frédéric Poulard, Les musées, les conservateurs et la mise en cuvre des politiques culturelles, op. cit., p. 115. 
La solution serait sans doute de récompenser financièrement les «bons élèves", et de conditionner le respect des impératifs de gestion des collections à l'octroi de subventions de fonctionnement, par exemple. Cependant, les moyens du service des musées de France sont, en la matière, limités, et le contexte actuel de crise des finances publiques ne permet pas d'envisager la réalisation d'une telle préconisation ${ }^{135}$.

Face à ces difficultés, l'État n'a pas d'autres moyens que de prêcher la pédagogie plutôt que d'user de la contrainte. C'est bien ce qui ressort du rapport précité sur la gestion des collections et des réserves des musées: aucune « sanction » apparente n'est envisagée, mais beaucoup d'interrogation sur la formation du personnel de conservation, le développement d'outils informatiques destinés à améliorer la "performance » des musées, etc.

Docteur en droit, élève avocate

135. Le rapport de la commission des affaires culturelles et de l'éducation de l'Assemblée nationale (précité), préconise de demander aux propriétaires des collections de renforcer ponctuellement les moyens financiers alloués au récolement... Or, si les services de l'État eux-mêmes doivent faire face à une crise des finances publiques sans précédent, celle-ci vise également les collectivités territoriales. On voit mal comment les collectivités territoriales pourraient, quant à elles, renforcer le financement de leurs musées. Voir Isabelle Attard, Michel Herbillon, Michel Piron et Marcel Rogemont, Rapport sur la gestion des réserves et des dépôts des musées, op. cit., p. 35-36. Voir également Didier Ryckner, "Un rapport parlementaire sur les musées ", La Tribune de l'art, 9 juillet 2014, disponible en ligne à l'adresse suivante: http://www.latribunedelart. com/un-rapport-parlementaire-sur-les-musees (consulté le 5 novembre 2015) et "Un rapport parlementaire sur les musées (2)», La Tribune de l'art, 24 décembre 2014, accessible à l'adresse suivante: http://www.latribunedelart.com/ un-rapport-parlementaire-sur-les-musees-suite (consulté le 5 novembre 2015). 